The AstrophysicAl JournaL, 331:746-763, 1988 August 15

(C) 1988. The American Astronomical Society. All rights reserved. Printed in U.S.A

\title{
MULTIFREQUENCY OBSERVATION OF THE OPTICALLY VIOLENT VARIABLE QUASAR 3C $446^{1}$
}

\author{
Joel N. Bregman, ${ }^{2}$ A. E. Glassgold, P. J. Huggins, and A. L. Kinney \\ New York University \\ I. MCHARDY \\ University of Leicester \\ J. R. Webb, J. T. Pollock, R. J. Leacock, A. G. Smith, and A. J. Pica \\ Rosemary Hill Observatory, University of Florida \\ H. D. Aller, M. F. Aller, ANd P. E. Hodge \\ University of Michigan Radio Astronomy Observatory \\ J. S. Miller and S. A. Stephens \\ Lick Observatory \\ W. A. Dent, T. J. BALONEK, AND R. BARVAinis ${ }^{2}$ \\ University of Massachusetts \\ G. Neugebauer, C. D. Impey, B. T. Soifer, K. Matthews, and J. H. Elias \\ Palomar Observatory, California Institute of Technology \\ AND \\ W. Z. WIŚNIEWSKI \\ Steward Observatory, University of Arizona \\ Received 1987 July 20 ; accepted 1988 February 2
}

\begin{abstract}
Extensive optical and radio monitoring data and seven multifrequency spectra were obtained of the violently variable quasar $3 \mathrm{C} 446$. The monitoring data suggest a correlation between the radio and optical outbursts, with the optical flare preceding the radio activity by 400-600 days. A difference in the statistical behavior of the optical and radio variability indicates that considerable processing occurs to the optical emitting plasma before it becomes radio emitting plasma. Within the radio band, outbursts proceed from high to low frequencies. An outburst in 1983 showed greater and more rapid variation in the optical than in the near-IR region. The 10-100 $\mu \mathrm{m}$ fluxes did not follow the higher frequency variation, suggesting a time delay between these spectral domains. During another time, the X-ray emission varied on a time scale of days and more rapidly than the UV or optical emission. On a time scale of weeks-months, the X-ray fluxes are well correlated with the UV-IR fluxes but not with the radio fluxes.

The multifrequency data show that the flat radio spectrum turns over at $3-10 \times 10^{11} \mathrm{~Hz}$ and the continuum steepens with frequency; $\alpha(\mathrm{IR})=-1.1, \alpha($ opt-UV) $=-2$ to -3 . The X-ray emission lies an order of magnitude above an extrapolation of the optical-UV spectrum and has a harder spectrum. The power is primarily concentrated in the submillimeter and infrared region. When the source is faint, a blue bump may be present. The flux in the $\mathrm{Ly} \alpha$ line is proportional to the UV continuum flux density when the source is bright but is independent of the continuum level when the source is faint.

The data suggest that the $\mathrm{X}$-rays are produced by the inverse Compton process from an emitting region $\left(10^{16} \mathrm{~cm}\right)$ smaller than but related to the synchrotron emitting UV-IR region. The characteristic size of the emitting region increases with decreasing frequency from $10^{16}$ (X-ray region) to $1-3 \times 10^{17} \mathrm{~cm}$ (far IRsubmillimeter region) to $10^{19}-10^{20} \mathrm{~cm}$ (radio region). Plasma conditions are best constrained at the frequency when the source becomes transparent, the far IR-submillimeter band, where $B \approx 3-100 \mathrm{G}, n \approx 40-100 \mathrm{~cm}^{-3}$; and the Doppler boosting factor $\delta \approx 1-5$.
\end{abstract}

Subject headings: infrared: sources — quasars — radiation mechanisms — radio sources: variable $\mathrm{X}$-rays: sources

\section{INTRODUCTION}

This paper is one in a series whose goal is to understand the continuum emission from violently variable quasars and $\mathrm{BL}$ Lacertae objects. Our approach is to study individual objects in depth by obtaining a series of "time-frozen" multifrequency

\footnotetext{
${ }^{1}$ In view of the large number of collaborators that are required for multifrequency programs, we list the authors by research groups, but we emphasize that each group has made important contributions to the data and analysis of this study.
}

spectra as well as using the results of long-term monitoring efforts. These fundamental data allow us to determine the relationship between emission in the different spectral domains and to apply theoretical models to the data to determine the physical conditions in the emitting plasma. Previous papers have discussed the properties of the X-ray bright BL Lacertae

${ }^{2}$ Work also carried out at the National Radio Astronomy Observatory, which is operated by Associated Universities, Inc., under contract with the National Science Foundation. 
object I Zw-187 (Bregman et al. 1982), the variable BL Lacertae object 0735+178 (Bregman et al. 1984), the Red Quasar 1413 + 135 (Bregman et al. 1981), the OVV 1156+295 (Glassgold et al. 1983; Wills et al. 1983), which was observed during a major flare, and the superluminal OVV 3C 345 (Bregman et al. 1986a). One result of these investigations is that the size and volume of the emitting region change as a function of frequency from the $\mathrm{X}$-ray through the radio band. Another result is that there is no simple relationship between the variation in the X-ray region and that of other wavebands. The source $3 \mathrm{C} 466$ provides an excellent opportunity to examine these issues in more depth.

Although 3C 466 is a typical blazar, with a steep, polarized optical continuum (Moore and Stockman 1984), polarized radio emission (Aller et al. 1985), and variability at all measured wavelengths, it is unusual in several respects. It is one of the most distant and luminous blazars known $(z=1.404)$, and it has an unusually steep optical spectrum. It is one of the only luminous sources (and the only blazar) in which the emission lines have been observed to vary in response to the continuum (Stephens and Miller 1984; Bregman et al. 1986b). The small equivalent widths of the optical lines led to the suggestion (Miller and French 1978) that it was a transition object between the BL Lacertae and the OVV classes. However, the weak emission lines are due to a paucity of ionizing photons and, relative to the number of ionizing photons, the emission line strengths are perfectly normal (Bregman et al. 1986b); henceforth, it will be referred to as an OVV. Because the 3C survey objects were the first quasars identified, 3C 446 has been studied at many wavebands for nearly twenty years, leading to an extensive data base.

The radio structure of $3 \mathrm{C} 446$ has been studied on the arcsecond scale with the VLA and on the milliarcsecond scale with VLBI. All structure is smaller than $2^{\prime \prime}$, with the most prominent VLA feature being an asymmetric 1 " extension that lies along the magnetic field direction (Brown et al. 1981). The VLBI studies indicate that there are several components (Simon, Johnston, and Spencer 1985): a core $<5$ mas that is brightest above 7-10 GHz, a 6-10 mas outer core, and a striking jet 250 mas in length (the position angle of the jet differs from that of the arcsecond structure by about $130^{\circ}$ ). Modeling of two VLBI data sets (Brown et al. 1981) suggests superluminal motion, although phase closure measurements are needed to verify this. Simon, Johnston, and Spencer (1985) have argued that bulk relativistic motion of the emitting plasma is likely, with a Dopper factor of $\delta>6$.

In this paper we present both monitoring and multifrequency observations of $3 \mathrm{C} 446$. The optical and radio monitoring span more than 15 years and allow us to search for long-term relationships between these wave bands ( $\S \mathrm{II} a$ ). The multifrequency spectra were obtained during an especially active period (1980-1985) and include data from many groundbased observatories as well as the Einstein Observatory, IRAS, $I U E$, and EXOSAT ( $(\mathrm{II} b)$. Further observations of Ly $\alpha$ line variation are discussed (§ IIc). The extensive continuum data are shown to provide important constraints for the theoretical models (§ III).

\section{OBSERVATIONS AND RESULTS}

\section{a) Monitoring Programs}

Nearly two decades of radio and optical monitoring data are available for $3 \mathrm{C} 446$, during which time several outbursts occurred. In 1983, an outburst occurred that was observed not only in the radio and optical wavebands, but also in the infrared region by $I R A S$. In the following paragraphs we describe the long-term monitoring and the 1983 outburst.

\section{i) Long-Term Monitoring}

The data presented in Figure 1 comprise the following: optical monitoring at $B(0.44 \mu \mathrm{m})$ from the Rosemary Hill Observatory of the University of Florida by A. Smith and collaborators; radio monitoring at 2, 4, and $6 \mathrm{~cm}(4.8,8,15.4$ $\mathrm{GHz}$ ) by $\mathrm{H}$. Aller and collaborators with the University of Michigan Radio Observatory; radio monitoring primarily at 6 $\mathrm{cm}, 1 \mathrm{~cm}$, and $3 \mathrm{~mm}(15.5,33.4$, and $89.6 \mathrm{GHz})$ by W. Dent and collaborators at Haystack Observatory and NRAO. The correlation between the optical and radio variability, first suggested by Balonek (1982), is more firmly established when recent data is included. The most complete sampling is at centimeter wavelengths, where the variation is greater at shorter wavelengths. The flux increases at $2 \mathrm{~cm}$ generally precede those at $4 \mathrm{~cm}$ by about $2-5$ months and at $6 \mathrm{~cm}$ by about $5-10$ months. Although the less frequent sampling at $3 \mathrm{~mm}$ (and 0.44 $\mu \mathrm{m})$ makes similar comparisons less precise, the $3 \mathrm{~mm}$ flux density variations are greater in amplitude and precede those at $2 \mathrm{~cm}$ by a fraction of a year. The minimum time scale of variation $(d t / d \log F)$ is given in Table 1 and the associated range of variation in the radio region is $\left(F_{\max } / F_{\min }\right)$ is 3.5 at 3 $\mathrm{mm}, 2.9$ at $2 \mathrm{~cm}, 2.3$ at $6 \mathrm{~cm}$, and 1.6 at $6 \mathrm{~cm}$. The trend of greater and more rapid variation at the shortest wavelengths, with events first appearing at the shortest wavelengths is consistent with models in which optical depth effects play a role.

The total flux variations at optical wavelengths $\left(F_{\max } / F_{\min }=40\right)$ are more than an order of magnitude greater than those observed at radio wavelengths, and the time scale for variation is much shorter (about a month). The optical variation, when averaged into 4 month bins (dashed line in Fig. 1), bears similarities to the radio variation. A correlation between the optical $(0.44 \mu \mathrm{m})$ and radio monitoring data $(2 \mathrm{~cm})$ was made to examine quantitatively whether optical outbursts are later manifest as radio flares. The data sets were shifted relative to each other and a radio flux was found for each optical datum, usually by interpolating between data points. The mean variance between the optical and radio data were calculated for a range of time shifts (Fig. 2; a positive time shift corresponds to the optical variation preceding the radio variation). We find a statistical significant minimum near 500 days, which is the time scale that the optical variation precedes the radio variation.

A comparison of the nature of the variability in the optical and radio bands can also be quantified. The behavior of varia-

TABLE 1

VARIABILITY TIME SCALES FOR 3C 446

\begin{tabular}{lcc}
\hline \hline Wavelength & $d t / d \ln F_{v}$ & $c d t / d \ln F_{v}(\mathrm{pc})$ \\
\hline $6 \mathrm{~cm} \ldots \ldots \ldots$ & $2.0 \mathrm{yr}$ & 0.61 \\
$4 \mathrm{~cm} \ldots \ldots \ldots$ & $1.5 \mathrm{yr}$ & 0.45 \\
$2 \mathrm{~cm} \ldots \ldots \ldots$ & $1.1 \mathrm{yr}$ & 0.34 \\
$3 \mathrm{~mm} \ldots \ldots .$. & 6 months & 0.15 \\
$10 \mu \mathrm{m} \ldots \ldots$. & $1-2$ months & 0.038 \\
$2.2 \mu \mathrm{m} \ldots \ldots$. & 20 days & 0.017 \\
$1.25 \mu \mathrm{m} \ldots \ldots$. & 17 days & 0.014 \\
$0.44 \mu \mathrm{m} \ldots \ldots$. & 10 days & 0.0083 \\
$1 \mathrm{keV} \ldots \ldots$. & 6 days & 0.0050 \\
\hline
\end{tabular}




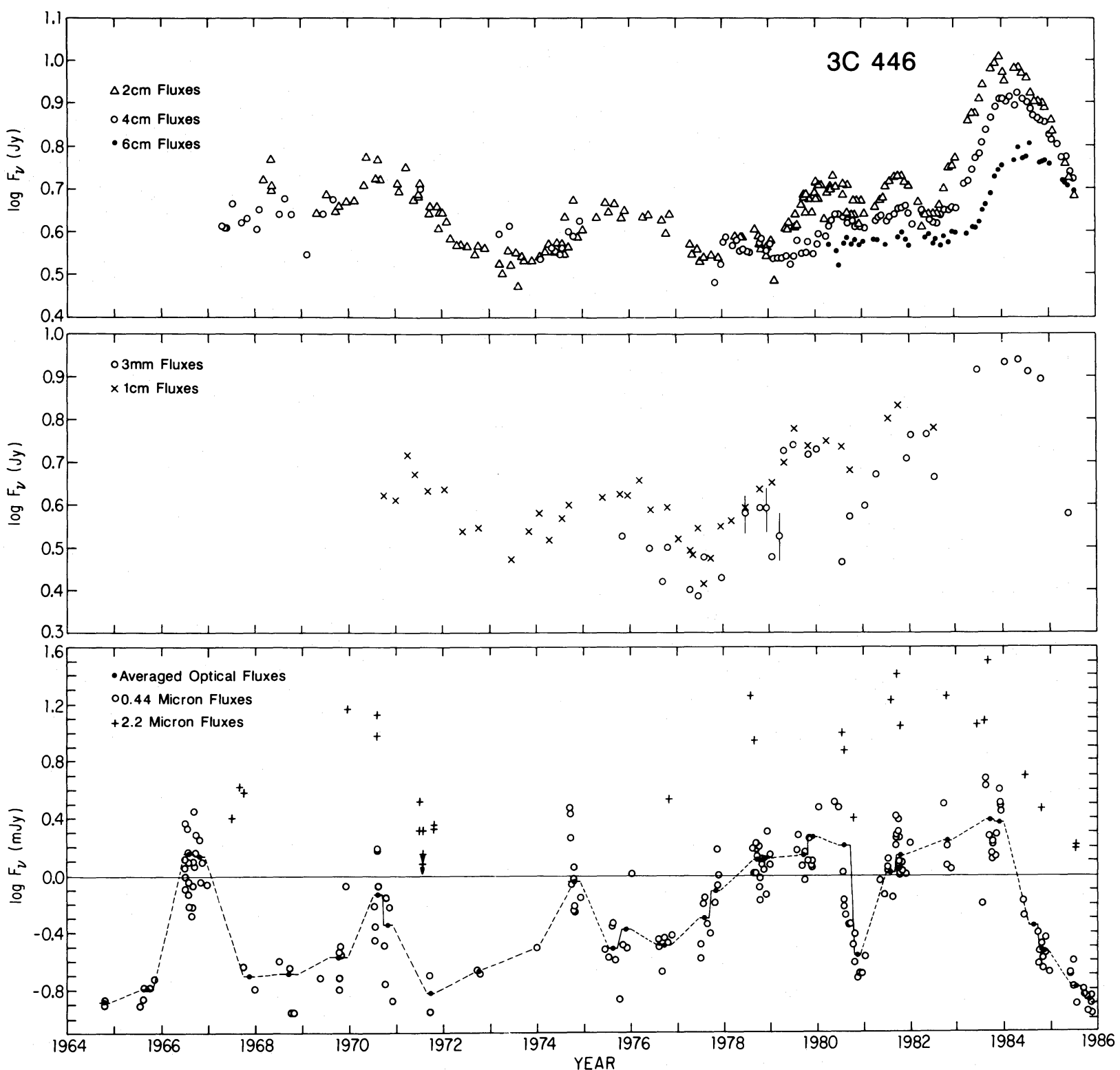

FIG. 1.-Radio and optical monitoring flux measurements of $3 \mathrm{C} 446$. The errors in the radio fluxes are generally less than a few percent and are not shown; errors less than $10 \%$ in other data are not displayed. The uncertainty in an individual optical flux measurement is $10 \%-20 \%\left(0.04-0.08\right.$ in log $\left.F_{\mathrm{v}}\right)$. For comparison with the radio data, the optical flux were binned in 4 month intervals. Points within a single observing season are connected with a solid line, and points in different seasons are connected with a dashed line.

bility data can be analyzed by Fourier techniques, or alternatively, by a structure function analysis (e.g., Simonetti, Cordes, and Heeschen 1985). The structure function shown in Figure 3 is the mean deviation $(S F)$ for data points separated by time $(\Delta t)$ :

$$
S F(\Delta t)=\left\langle\left[F_{v}(t)-F_{v}(t+\Delta t)\right]^{2}\right\rangle .
$$

The radio data have been divided in half (in time) and treated separately. The differences in the two samples at the longest $\Delta t$ indicate that all relevant time scales have not yet been seen. The radio data show little variation at time scales less than a month, but for $\Delta t>1$ month, the slopes of the structure function are $a=0.90 \pm 0.07$ [sample 1 , where $S F \propto(\Delta t)^{a}$ ] and $a=1.2 \pm 0.05$ (sample 2). When all of the radio data are analyzed as a single sample, $a=1.04 \pm 0.06$, and a flattening is suggested for $\Delta t>4 \mathrm{yr}$. The optical data shows $a=0.6 \pm 0.1$ for $\log \Delta t$ (days) $<2.3$ and $a \approx 0.0$ for $\log \Delta t$ (days) $>2.3$; there is no difference in the results when the optical data are divided into two samples. The difference in the structure function indicates that there is a significant difference in the variability behavior of the radio and optical bands. Consequently, if optically emitting plasma eventually becomes radio emitting plasma, significant processing of the plasma must occur.

For the range of interest, the slope $a$ corresponds to a power spectrum $P(\omega) \propto \omega^{-(a+1)}$, so the radio data behave like shot noise $(a=1)$ when 1 month $<\Delta t<4$ yr while the behavior of the optical data is between flicker noise $(a=0)$ and shot noise when $\Delta t<1 \mathrm{yr}$. 


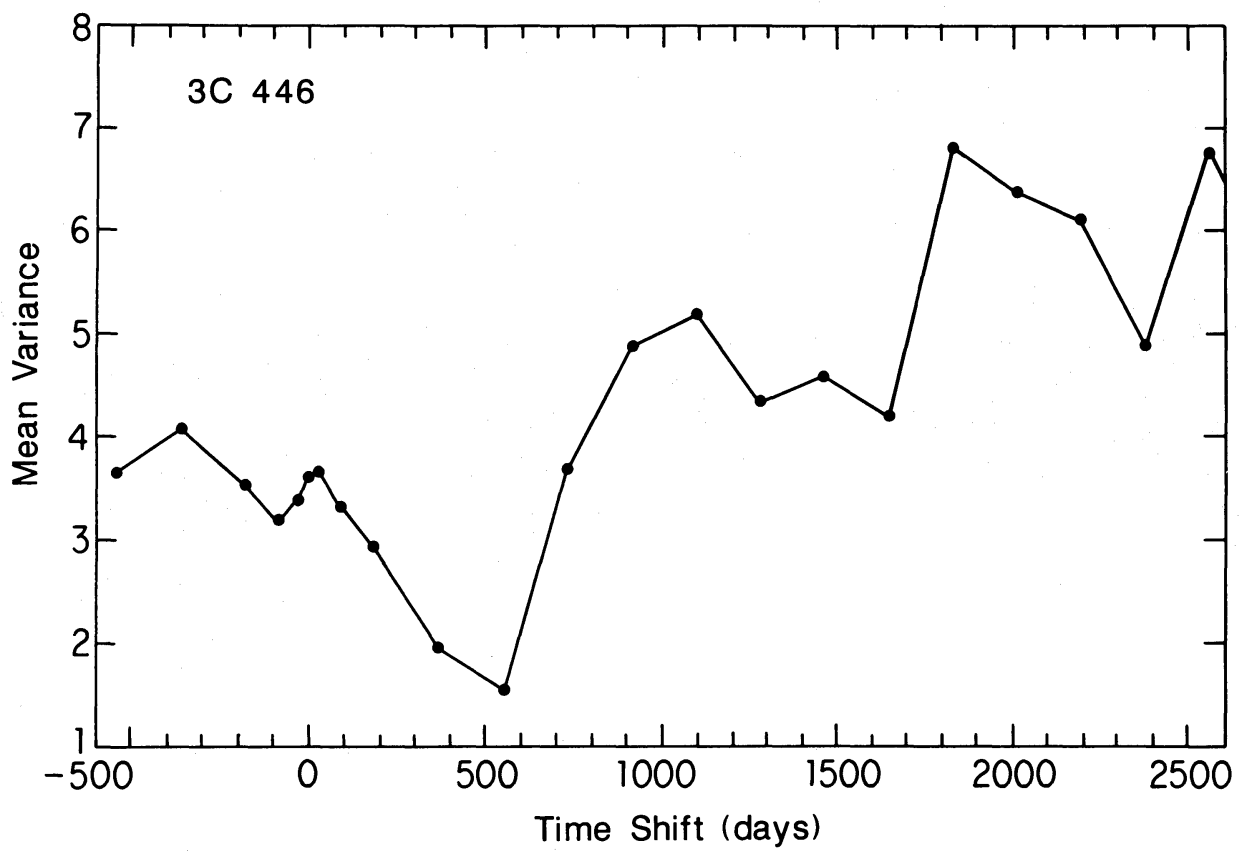

Fig. 2.-The mean variance between the optical and the $2 \mathrm{~cm}$ data are shown as a function of time delay between the data sets. The statistically significant minimum near 500 days shows that optical variations are correlated with and precede the radio variation.

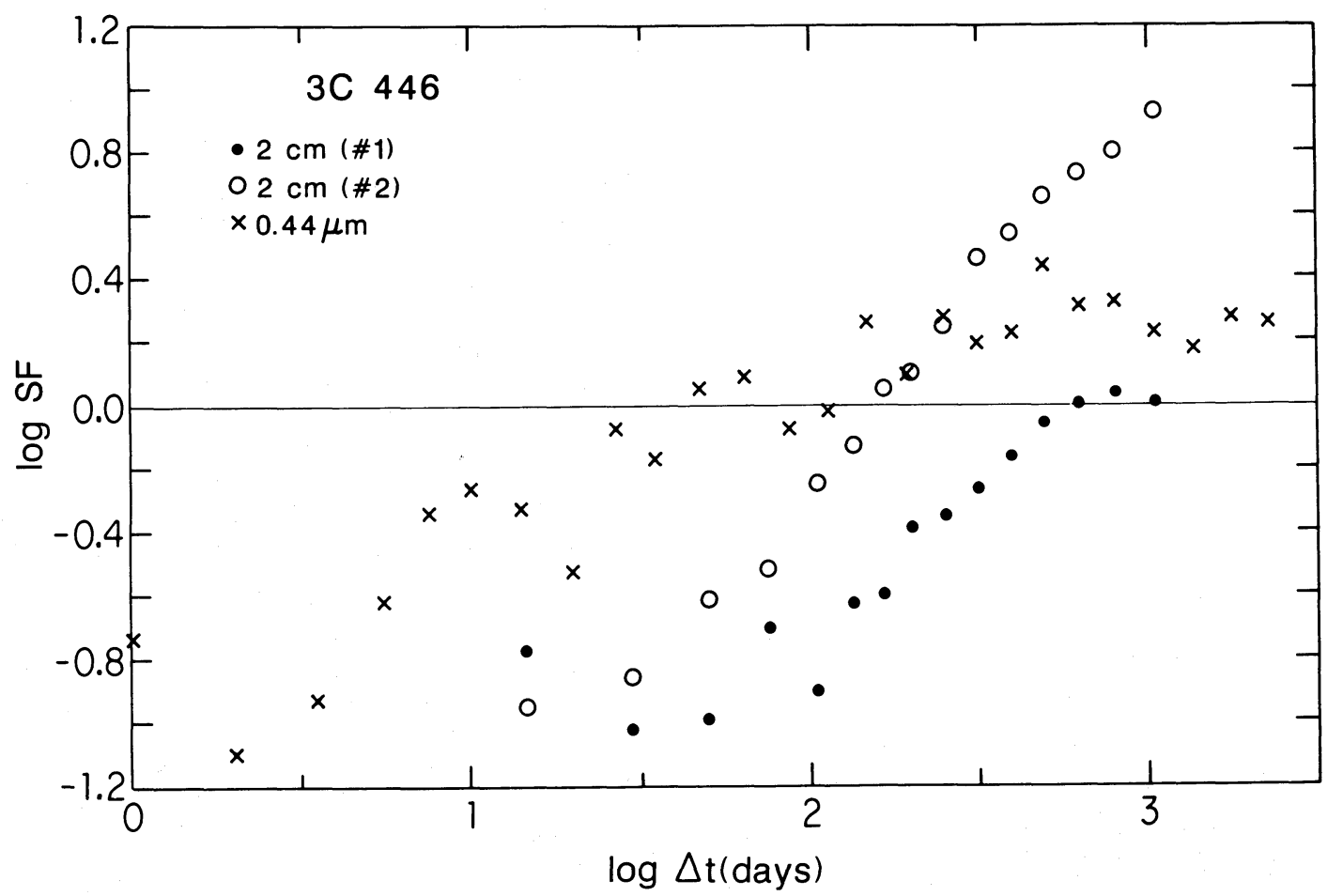

FIG. 3.-The structure function $(S F)$ for the optical and the radio monitoring data. The radio data are divided in time and somewhat different structure functions result, indicating that not all timescales have been seen. The structure function for the optical data is significantly different from that of the radio data. 
ii) The 1983 Outburst

The 1983 observing season provided an unusual opportunity to study flux variation in the optical, nearinfrared, and infrared spectral regions. Figure 4 represents the results of optical monitoring observations taken at Rosemary Hill and by Barbieri et al. (1985), infrared observations by

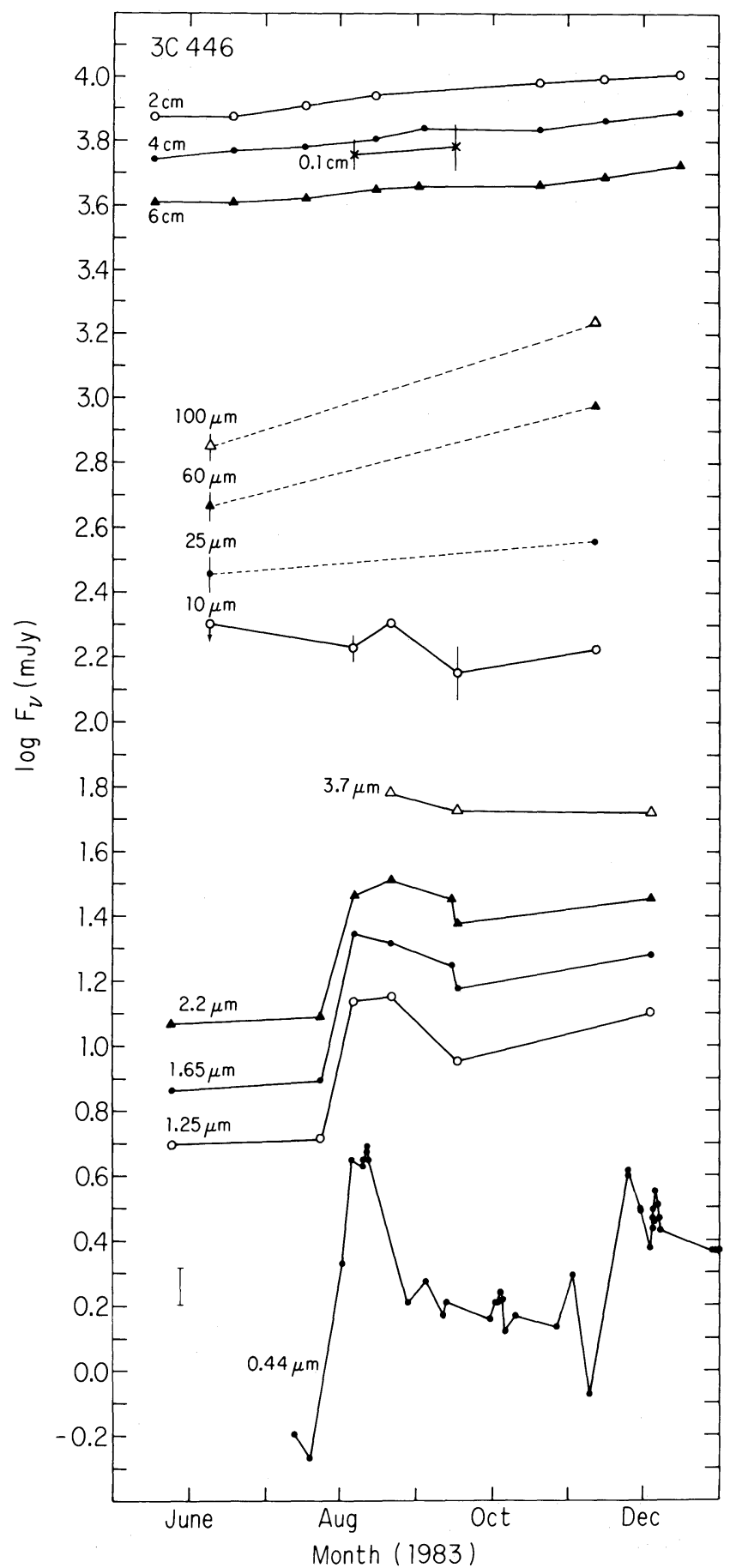

Fig. 4.-Variation of 3C 446 during the period 1983 June-December. Errors in the fluxes of less than $10 \%$ are not displayed. Dashed lines that connect the 25,60 , and $100 \mu$ m fluxes are not meant to imply continuous flux variations. The variability amplitude increases and the time scale decreases with increasing frequency.
Neugebauer et al. (1986), Brown et al. (1986), and T. Roellig (this paper); infrared measurements using IRAS by Neugebauer et al. (1986); submillimeter data from Brown et al. (1986); and radio flux densities from UMRAO. During this time frame, optical flux variations were dramatic and rapid, such as the three-week flare in August. A flare was also seen in the near-infrared region, although the amplitude decreases with increasing wavelength from 0.44 to $10 \mu \mathrm{m}$. The data suggest that flux variation develops more slowly with increasing wavelength. In November the source brightened again in the optical region.

The brightness increases at IRAS wavelengths, first discussed by Neugebauer et al. (1986), are similar at $100 \mu \mathrm{m}$ and $60 \mu \mathrm{m}$, but the increase observed at $25 \mu \mathrm{m}$ is smaller (the ratio of the November fluxes to the June fluxes is $2.5 \pm 0.3$, $2.0 \pm 0.2$, and $1.3 \pm 0.2$ for $100 \mu \mathrm{m}, 60 \mu \mathrm{m}$, and $25 \mu \mathrm{m})$. It is impossible to determine whether the lack of brightening at 25 $\mu \mathrm{m}$ is real or was missed due to the infrequent sampling. One possible scenario is that the outburst observed at optical wavelengths during August occurred later at longer wavelengths. The millimeter and centimeter data show little indication of the violent activity seen in the near infrared and optical region.

These 1983 variability data permit one to test whether synchrotron emission models can reproduce the characteristics of the outburst. The flux decrease of the August flare event is greater and apparently faster at the shortest wavelength. A measure of the decrease in the flux densities between August and September for $0.55-10 \mu \mathrm{m}$ is given in Figure 5. Such behavior was suggested by Kardashev (1962) and others as the result of high-energy electrons losing their energy more rapidly than lower energy electrons (via synchrotron losses). We have calculated the radiation from an ensemble of electrons with an initial power-law distribution and in which the pitch angle is preserved as the electrons lose energy through synchrotron emission (Kardashev 1962; Pacholczyk 1970; Meyers and Spangler 1985). This model leads to a cutoff in the electron spectrum that is sharper than other models involving synchrotron losses (e.g., when pitch angle isotropy is preserved). The postflare data are just barely consistent with this extreme model with $B \approx 0.1 \mathrm{G}$ (Fig. 5), but the data could be better fitted if the cutoff to the synchrotron emission were even steeper.

We have developed a simple model that provides a good fit to the data and in which synchrotron losses are not a determining factor. We consider a power-law electron spectrum with an abrupt cutoff at some energy; this provides a sharp spectral cutoff. If a plasma with this electron distribution flows through regions of varying magnetic field, the shape of the spectrum is preserved as it shifts to higher (or lower) frequency and flux density. The resulting flux density variation has been fit to the data in Figure 5. This model, in which one allows for variation in the magnetic field, and possibly the total particle density while preserving the truncated electron spectrum, can also account for the behavior seen during the onset of the flare. An increase in the magnetic field serves to shift the preexisting spectrum to higher frequencies and greater flux density. Because of the rapid steepening in the optical region, shifting the spectrum to higher frequency leads to an increase that is greater in the optical than in the infrared region. The postflare behavior is explained in the opposite manner by a decrease of the magnetic field in the emitting region. Unlike the synchrotron loss model, this model does not lead to a value for the magnetic field. 


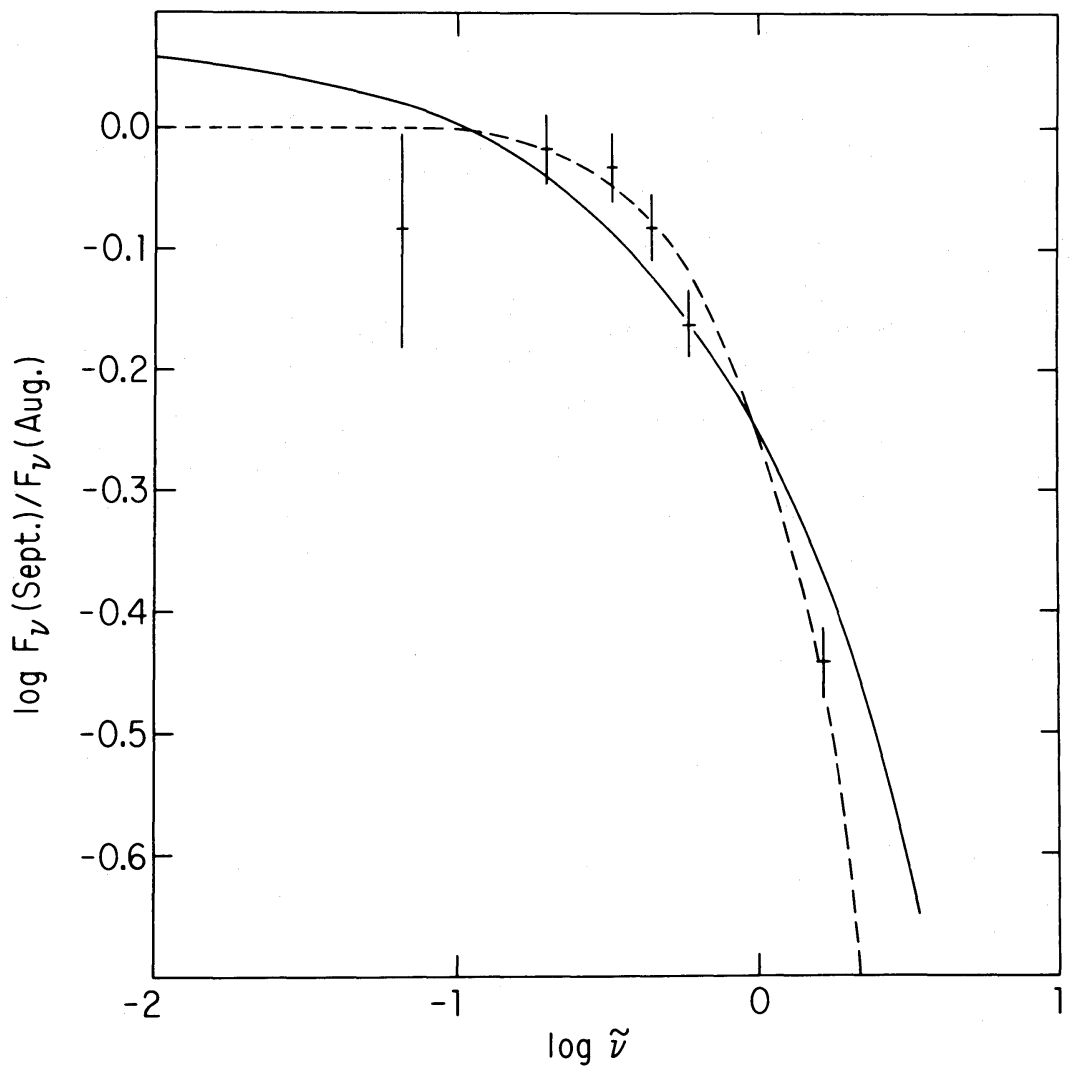

Fig. 5.-The flux decrease between 1983 September and August are measured by the ratio of the fluxes at 10, 3.7, 2.2, 1.65, 1.25, and $0.44 \mu \mathrm{m}$ (crosses). Although the relative frequency spacing has been preserved, the data are plotted in terms of a dimensionless frequency for comparison with theoretical predictions. The solid line is the prediction from a model in which synchrotron losses are important. A better fit is provided by a model without synchrotron losses, but with plasma parameters that change with time (dashed line).

\section{b) Multifrequency Observations}

A series of seven multifrequency observations were obtained during 1980-1985, a time of unusual radio and optical variation for 3C 446 (Fig. 1; Table 2). The most complete multifrequency spectrum was obtained during 1983 December and is discussed first. The other multifrequency spectra are analyzed relative to the 1983 December spectrum. All data in Figs. 6-8 have been dereddened by $E(B-V)=0.05 \mathrm{mag}$ (Burstein and Heiles 1980) or $N_{\mathrm{H}}=6 \times 10^{20} \mathrm{~cm}^{-2}$ when necessary.

\section{i) 1983 December Spectrum}

The 1983 December spectrum was obtained near the peak of a major radio and optical outburst that lasted for about a year. In this spectrum (Fig. 6), the flat radio spectrum turns over in the submillimeter range $(\log v=11.5-12)$ and becomes progressively steeper with increasing frequency. The slope in the different bands are as follows: $\alpha=-1.08$ in the infrared region (3-100 $\mu \mathrm{m}), \alpha=-1.37 \pm 0.05$ at $1.25 \mu \mathrm{m}, \alpha=-1.67 \pm 0.05$ at $0.44 \mu \mathrm{m}$, and $\alpha=-2.5 \pm 0.3$ in the ultraviolet region.

An extrapolation of the infrared-ultraviolet continuum to higher frequencies falls about an order of magnitude below the X-ray data in the 1983 December spectrum as well as in other multifrequency spectra, such as the 1980 June spectrum, where the X-ray flux is accurately known. This indicates that the $X$-rays are not just an extension of the infrared-ultraviolet synchrotron emission, but arise either from some other process (e.g., inverse Compton emission), or from the synchrotron process in a different spatial location.

Limited spectral information is contained in the X-ray data. The $0.04-2 \mathrm{keV}$ range of the EXOSAT low-energy imaging instrument (LE; CMA) was divided up into two energy bands with the AlP and $3 \mathrm{Lx}$ filters. The source was also detected in the medium-energy instrument (ME) in the 2-6 keV window. Combining these data produces a spectral index of $\alpha=-0.8$. The statistical uncertainty in this value is 0.2 , but systematic errors may increase it to as much as 0.4 . A spectral index was also determined from the Einstein IPC data (0.1-4.5 keV), and Garilli and Tagliaferri (1986) found that the best data gave $\alpha=-0.9 \pm 0.7$ (nonsimultaneous data of 1979 December). Recent observations from the GINGA satellite in the $1-30 \mathrm{keV}$ band show a continuum slightly shallower than the EXOSAT and Einstein results (Makino 1987, private communication). These observations indicate that the $\mathrm{X}$-ray continuum is harder than the optical-ultraviolet continuum, which supports the suggestion that the $\mathrm{X}$-rays are produced by an emission process different from that responsible for the opticalultraviolet continuum.

Most of the power emitted emerges in the submillimeter through the optical region. If the X-ray continuum had a "universal" spectrum found for the brightest Seyfert galaxies $(\alpha=-0.65$ up to $165 \mathrm{keV}$; Rothschild et al. 1983$)$, the estimated integrated $\mathrm{X}$-ray power is less than the integrated submillimeter-optical power by a factor of 4 . As X-ray spectra 
TABLE 2

3C 446 MULTIFREQUENCY DATA

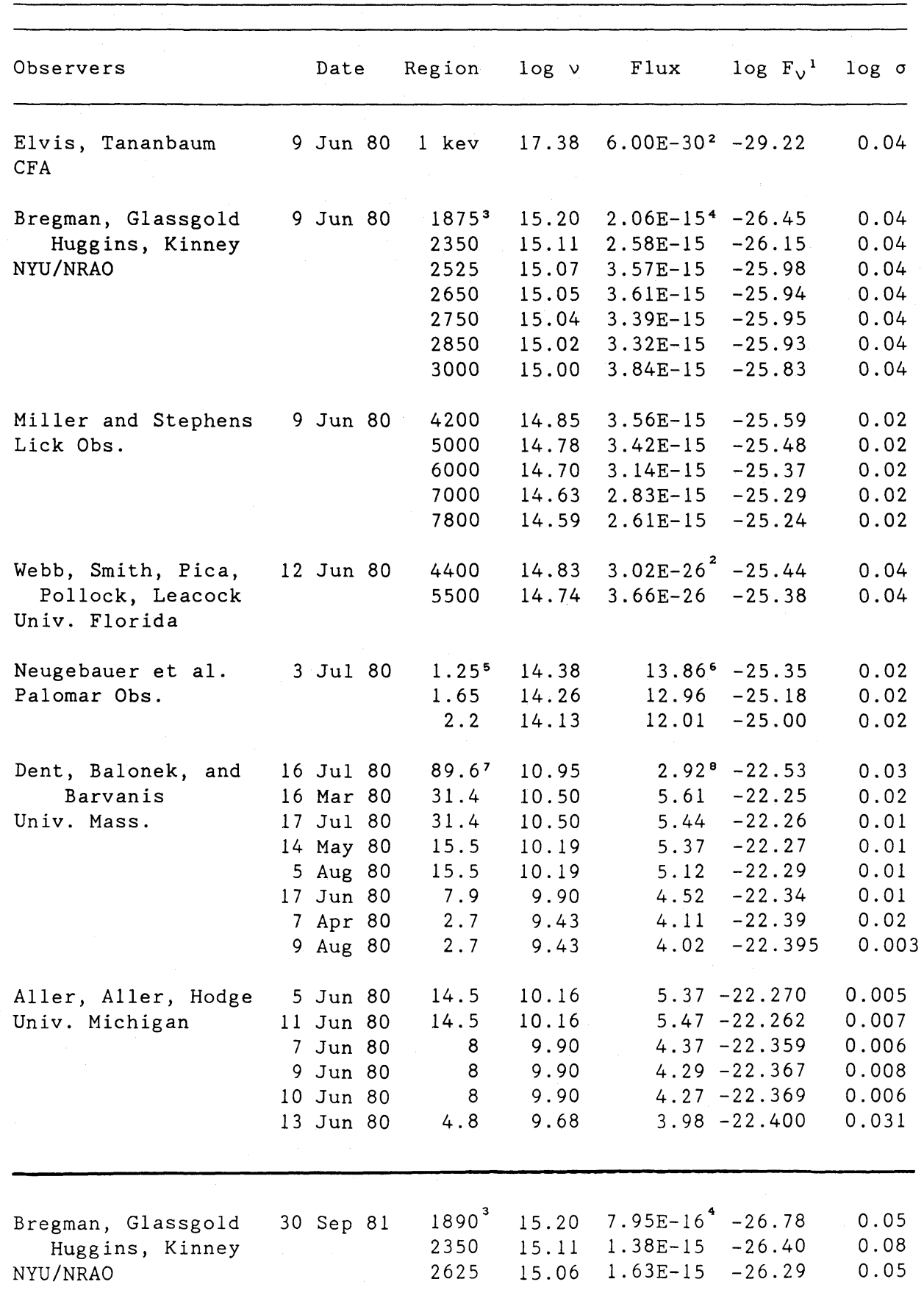

of OVVs and BL Lacertae objects seem to be steeper than that of bright Seyferts, the total X-ray power is probably less (Urry 1986).

\section{ii) Other Multifrequency Spectra}

To highlight the differences between multifrequency spectra, the other spectra are displayed relative to the 1983 December data in Figure 7. A fifth-order polynomial was fitted to the 1983 infrared through ultraviolet data and all spectra were divided by this function. In the radio and $\mathrm{X}$-ray regions, the values (or averages in some cases) were divided into the other spectra; an extrapolation was required for the value at $33 \mathrm{GHz}$ for the 1980 June and 1981 October data.

The residual between the observations and the fifth-order fit to the 1983 December radio spectrum is generally negligible (standard deviation $<0.02$ in the $\log$ of the flux ratios), while the residuals in the infrared and optical regions are larger than the statistical errors and reflect calibration difficulties (typically 0.05 in the $\log F_{y}$ and for a few points, as high as 0.15$)$; the $\mathrm{X}$-ray data are discussed below. The residuals provide an estimate of the uncertainties involved in obtaining multifrequency 
TABLE 2-Continued

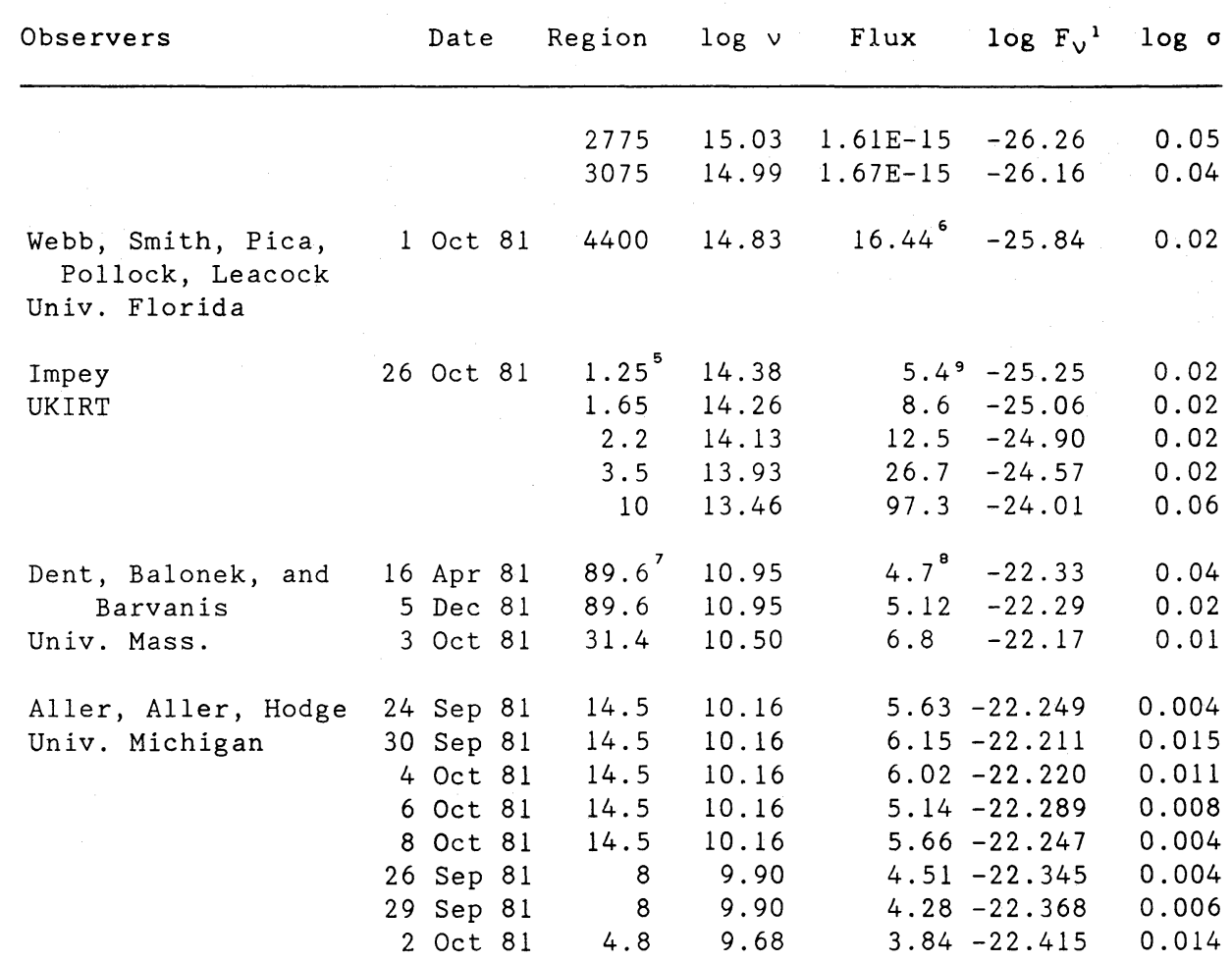

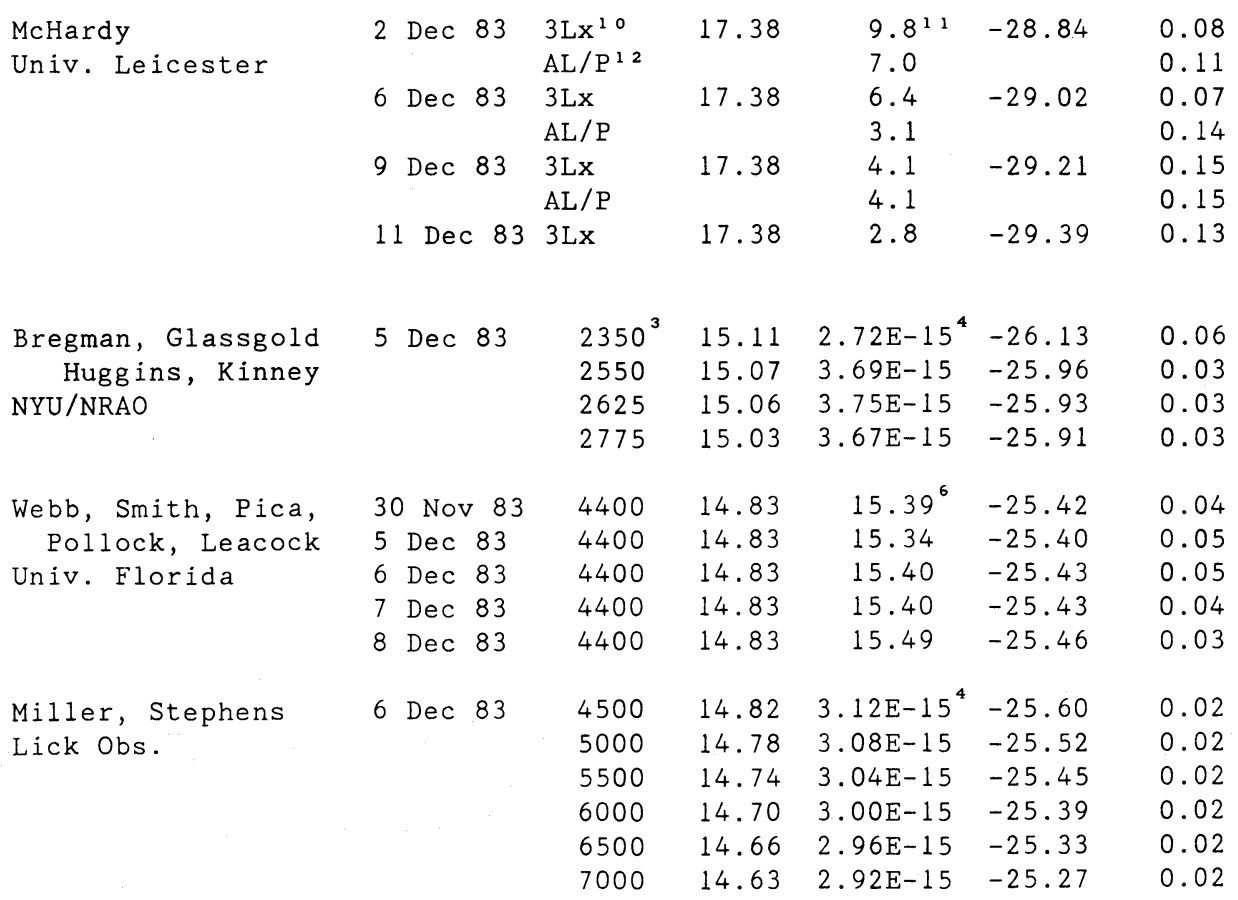


TABLE 2-Continued

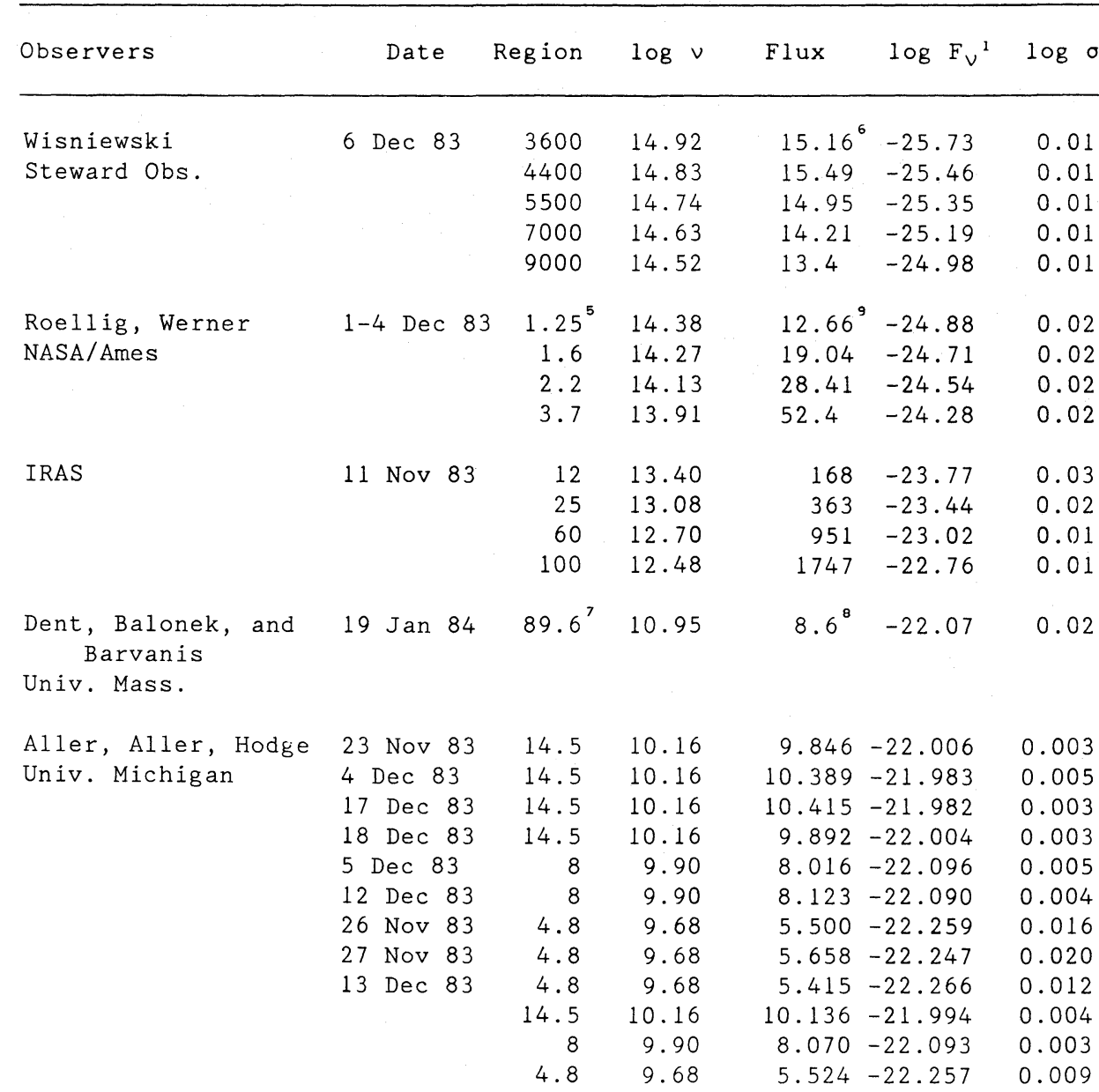

\begin{tabular}{|c|c|c|c|c|c|c|}
\hline $\begin{array}{l}\text { McHardy } \\
\text { Univ. Leicester }\end{array}$ & 31 May 84 & $3 L x^{10}$ & 17.38 & $2.3^{11}$ & -29.47 & 0.14 \\
\hline $\begin{array}{l}\text { Bregman, Glassgold, } \\
\text { Huggins, Kinney } \\
\text { NYU/NRAO }\end{array}$ & 29 May 84 & $\begin{array}{l}2350^{3} \\
2550 \\
2625 \\
2775\end{array}$ & $\begin{array}{l}15.11 \\
15.07 \\
15.06 \\
15.03\end{array}$ & $\begin{array}{l}9.00 E-16^{4} \\
5.26 E-16 \\
6.55 E-16 \\
8.81 E-16\end{array}$ & $\begin{array}{l}-26.61 \\
-26.80 \\
-26.69 \\
-26.53\end{array}$ & $\begin{array}{l}0.08 \\
0.07 \\
0.06 \\
0.03\end{array}$ \\
\hline $\begin{array}{l}\text { Webb, Smith, Pica, } \\
\text { Pollock, Leacock } \\
\text { Univ. Florida }\end{array}$ & $\begin{array}{l}31 \text { May } 84 \\
2 \text { Jun } 84\end{array}$ & $\begin{array}{l}4400 \\
4400\end{array}$ & $\begin{array}{l}14.83 \\
14.83\end{array}$ & $\begin{array}{l}17.50^{6} \\
17.32\end{array}$ & $\begin{array}{l}-26.27 \\
-26.20\end{array}$ & $\begin{array}{l}0.06 \\
0.06\end{array}$ \\
\hline $\begin{array}{l}\text { Miller, Stephens } \\
\text { Lick Obs. }\end{array}$ & 31 May 84 & $\begin{array}{l}4500 \\
5000 \\
5500 \\
6000 \\
6500 \\
7000\end{array}$ & $\begin{array}{l}14.82 \\
14.78 \\
14.74 \\
14.70 \\
14.66 \\
14.63\end{array}$ & $\begin{array}{l}1.02 E-15 \\
1.01 E-15 \\
9.90 E-16 \\
1.00 E-15 \\
9.60 E-16 \\
9.40 E-16\end{array}$ & $\begin{array}{l}-26.08 \\
-26.00 \\
-25.94 \\
-25.86 \\
-25.82 \\
-25.77\end{array}$ & $\begin{array}{l}0.02 \\
0.02 \\
0.02 \\
0.02 \\
0.02 \\
0.02\end{array}$ \\
\hline
\end{tabular}


TABLE 2-Continued

\begin{tabular}{|c|c|c|c|c|c|c|}
\hline Observers & Date & Region & $\log v$ & Flux & $\log F_{V}^{1}$ & $\log$ \\
\hline $\begin{array}{l}\text { Neugebauer } \\
\text { Palomar Obs. }\end{array}$ & 8 Jun 84 & $\begin{array}{l}1.3^{5} \\
1.65 \\
2.2\end{array}$ & $\begin{array}{l}14.36 \\
14.26 \\
14.13\end{array}$ & $\begin{array}{l}1.85^{9} \\
3.02 \\
5.08\end{array}$ & $\begin{array}{l}-25.72 \\
-25.51 \\
-25.29\end{array}$ & $\begin{array}{l}0.02 \\
0.02 \\
0.02\end{array}$ \\
\hline $\begin{array}{l}\text { Dent, O'Dea, Kinzel, } \\
\text { Barvanis } \\
\text { Univ. Mass. }\end{array}$ & 18 May 84 & $89.6^{7}$ & 10.95 & $8.69^{8}$ & -22.06 & 0.02 \\
\hline $\begin{array}{l}\text { Aller, Aller, Hodge } \\
\text { Univ. Michigan }\end{array}$ & $\begin{array}{l}28 \text { May } 84 \\
29 \text { May } 84 \\
2 \text { Jun } 84 \\
25 \text { May } 84 \\
1 \text { Jun } 84 \\
4 \text { Jun } 84 \\
30 \text { May } 84 \\
5 \text { Jun } 84\end{array}$ & $\begin{array}{r}14.5 \\
14.5 \\
14.5 \\
8 \\
8 \\
8 \\
4.8 \\
4.8\end{array}$ & $\begin{array}{r}10.16 \\
10.16 \\
10.16 \\
9.90 \\
9.90 \\
9.90 \\
9.68 \\
9.68\end{array}$ & $\begin{array}{l}9.38 \\
9.173 \\
9.476 \\
8.869 \\
7.907 \\
8.181 \\
6.117 \\
6.314\end{array}$ & $\begin{array}{l}-22.027 \\
-22.037 \\
-22.023 \\
-22.052 \\
-22.101 \\
-22.087 \\
-22.213 \\
-22.199\end{array}$ & $\begin{array}{l}0.006 \\
0.006 \\
0.003 \\
0.008 \\
0.006 \\
0.005 \\
0.013 \\
0.024\end{array}$ \\
\hline
\end{tabular}

\begin{tabular}{|c|c|c|c|c|c|c|c|c|}
\hline $\begin{array}{l}\text { McHardy } \\
\text { Univ. Leicester }\end{array}$ & 25 & Oct & 84 & $3 \mathrm{Lx}^{10}$ & 17.38 & $2.1^{11}$ & -29.51 & 0.18 \\
\hline $\begin{array}{l}\text { Bregman, Glassgold, } \\
\text { Huggins, Kinney } \\
\text { NYU/NRAO }\end{array}$ & 25 & Oct & 84 & $\begin{array}{l}2625^{3} \\
2775\end{array}$ & $\begin{array}{l}15.06 \\
15.03\end{array}$ & $\begin{array}{l}4.54 E-16^{4} \\
5.31 E-16\end{array}$ & $\begin{array}{l}-26.85 \\
-26.75\end{array}$ & $\begin{array}{l}0.06 \\
0.03\end{array}$ \\
\hline $\begin{array}{l}\text { Webb, Smith, Pica, } \\
\text { Pollock, Leacock } \\
\text { Univ. Florida }\end{array}$ & $\begin{array}{l}25 \\
26\end{array}$ & $\begin{array}{l}\text { Oct } \\
\text { Oct }\end{array}$ & $\begin{array}{l}84 \\
84\end{array}$ & $\begin{array}{l}4400 \\
4400\end{array}$ & $\begin{array}{l}14.83 \\
14.83\end{array}$ & $\begin{array}{l}18.19^{6} \\
17.96\end{array}$ & $\begin{array}{l}-26.54 \\
-26.45\end{array}$ & $\begin{array}{l}0.03 \\
0.04\end{array}$ \\
\hline $\begin{array}{l}\text { Miller, Stephens } \\
\text { Lick Obs. }\end{array}$ & 22 & Oct & 84 & $\begin{array}{l}4500 \\
5000 \\
5500 \\
6000 \\
6500 \\
7000 \\
7500\end{array}$ & $\begin{array}{l}14.82 \\
14.78 \\
14.74 \\
14.70 \\
14.66 \\
14.63 \\
14.60\end{array}$ & $\begin{array}{l}3.97 E-16^{4} \\
3.89 E-16 \\
3.93 E-16 \\
3.98 E-16 \\
3.91 E-16 \\
3.70 E-16 \\
3.69 E-16\end{array}$ & $\begin{array}{l}-26.49 \\
-26.42 \\
-26.34 \\
-26.26 \\
-26.21 \\
-26.17 \\
-26.12\end{array}$ & $\begin{array}{l}0.02 \\
0.02 \\
0.02 \\
0.02 \\
0.02 \\
0.02 \\
0.02\end{array}$ \\
\hline $\begin{array}{l}\text { Neuge bauer } \\
\text { Palomar Obs. }\end{array}$ & 80 & Oct & 84 & $\begin{array}{l}1.3^{5} \\
1.65 \\
2.2\end{array}$ & $\begin{array}{l}14.36 \\
14.26 \\
14.13\end{array}$ & $\begin{array}{l}1.29^{9} \\
2.05 \\
2.98\end{array}$ & $\begin{array}{l}-25.87 \\
-25.68 \\
-25.52\end{array}$ & $\begin{array}{l}0.25 \\
0.03 \\
0.07\end{array}$ \\
\hline Brown et al. (1986) & 14 & Oct & 84 & $\begin{array}{l}1.25 \\
1.65 \\
2.2 \\
3.5\end{array}$ & $\begin{array}{l}14.38 \\
14.26 \\
14.13 \\
13.93\end{array}$ & $\begin{array}{l}1.3 \\
2.48 \\
3.74 \\
11.2\end{array}$ & $\begin{array}{l}-25.87 \\
-25.60 \\
-25.42 \\
-24.95\end{array}$ & $\begin{array}{l}0.02 \\
0.02 \\
0.02 \\
0.11\end{array}$ \\
\hline $\begin{array}{l}\text { Dent, O'Dea, Kinzel, } \\
\text { Barvanis } \\
\text { Univ. Mass. }\end{array}$ & 23 & Oct & 84 & $89.6^{7}$ & 10.95 & 7.84 & -22.11 & 0.01 \\
\hline $\begin{array}{l}\text { Aller, Aller, Hodge } \\
\text { Univ. Michigan }\end{array}$ & $\begin{array}{l}16 \\
17\end{array}$ & $\begin{array}{l}\text { Oct } \\
\text { Oct }\end{array}$ & $\begin{array}{l}84 \\
84\end{array}$ & $\begin{array}{r}14.5 \\
8\end{array}$ & $\begin{array}{r}10.16 \\
9.90\end{array}$ & $\begin{array}{l}8.04 \\
7.3\end{array}$ & $\begin{array}{l}-22.094 \\
-22.136\end{array}$ & $\begin{array}{l}0.002 \\
0.003\end{array}$ \\
\hline
\end{tabular}


TABLE 2-Continued

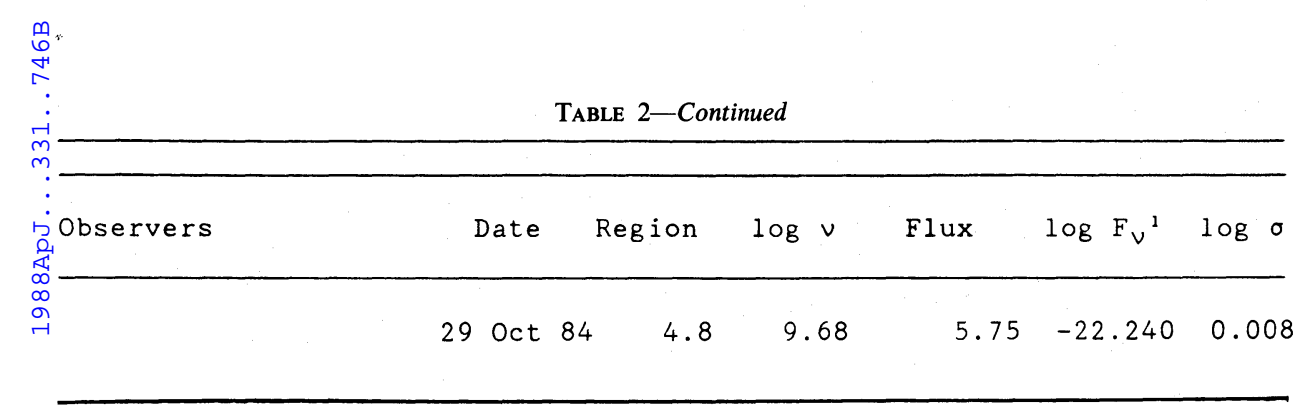

\begin{tabular}{|c|c|c|c|c|c|c|}
\hline McHardy & 28 May 85 & $3 L x^{10}$ & 17.38 & $1.2^{11}$ & -29.74 & 0.30 \\
\hline Univ. Leicester & $\begin{array}{l}6 \text { Jun } 85 \\
13 \text { Jun } 85\end{array}$ & $\begin{array}{l}3 L x \\
3 L x\end{array}$ & $\begin{array}{l}17.38 \\
17.38\end{array}$ & $\begin{array}{l}1.2 \\
1.2\end{array}$ & $\begin{array}{l}-29.74 \\
-29.74\end{array}$ & $\begin{array}{l}0.26 \\
0.20\end{array}$ \\
\hline $\begin{array}{l}\text { Bregman, Glassgold, } \\
\text { Hugg ins, Kinney } \\
\text { NYU/NRAO }\end{array}$ & 18 May 85 & $\begin{array}{l}2300^{3} \\
2625 \\
2775 \\
3075 \\
2300 \\
2625 \\
2775\end{array}$ & $\begin{array}{l}15.12 \\
15.06 \\
15.03 \\
14.99 \\
15.12 \\
15.06 \\
15.03\end{array}$ & $\begin{array}{l}3.80 \mathrm{E}-16^{4} \\
2.75 \mathrm{E}-16 \\
3.94 \mathrm{E}-16 \\
4.32 \mathrm{E}-16 \\
2.25 \mathrm{E}-16 \\
3.59 \mathrm{E}-16 \\
3.82 \mathrm{E}-16\end{array}$ & $\begin{array}{l}-26.98 \\
-27.06 \\
-26.87 \\
-26.76 \\
-27.22 \\
-26.95 \\
-26.88\end{array}$ & $\begin{array}{l}0.13 \\
0.08 \\
0.03 \\
0.06 \\
0.18 \\
0.06 \\
0.04\end{array}$ \\
\hline $\begin{array}{l}\text { Webb, Smith, Pica, } \\
\text { Pollock, Leacock } \\
\text { Univ. Florida }\end{array}$ & $\begin{array}{l}27 \text { May } 85 \\
29 \text { May } 85\end{array}$ & $\begin{array}{l}4400 \\
4400\end{array}$ & $\begin{array}{l}14.83 \\
14.83\end{array}$ & $\begin{array}{l}18.34^{6} \\
18.36\end{array}$ & $\begin{array}{l}-26.60 \\
-26.61\end{array}$ & $\begin{array}{l}0.05 \\
0.04\end{array}$ \\
\hline $\begin{array}{l}\text { UKIRT Service } \\
\text { Observing }\end{array}$ & 20 Jun 85 & $\begin{array}{l}1.25^{5} \\
1.65 \\
2.2 \\
3.5\end{array}$ & $\begin{array}{l}14.38 \\
14.26 \\
14.13 \\
13.93\end{array}$ & $\begin{array}{l}1.39^{9} \\
1.55 \\
2.05 \\
6.4\end{array}$ & $\begin{array}{l}-25.84 \\
-25.80 \\
-25.68 \\
-25.19\end{array}$ & $\begin{array}{l}0.02 \\
0.02 \\
0.02 \\
0.02\end{array}$ \\
\hline $\begin{array}{l}\text { Dent, O'Dea, Kinzel, } \\
\text { Barvanis } \\
\text { Univ. Mass. }\end{array}$ & 21 May 85 & $89.6^{7}$ & 10.95 & 3.79 & -22.42 & 0.02 \\
\hline $\begin{array}{l}\text { Aller, Aller, Hodge } \\
\text { Univ. Michigan }\end{array}$ & $\begin{array}{l}20 \text { May } 85 \\
11 \text { May } 85 \\
14 \text { May } 85 \\
9 \text { Jun } 85 \\
22 \text { Jun } 85 \\
5 \text { Jun } 85\end{array}$ & $\begin{array}{r}14.5 \\
8 \\
4.8 \\
14.5 \\
8 \\
4.8\end{array}$ & $\begin{array}{r}10.16 \\
9.90 \\
9.68 \\
10.16 \\
9.90 \\
9.68\end{array}$ & $\begin{array}{l}5.75 \\
5.93 \\
5.16 \\
5.31 \\
5.46 \\
5.08\end{array}-$ & $\begin{array}{l}-22.240 \\
-22.226 \\
-22.287 \\
-22.274 \\
-22.262 \\
-22.294\end{array}$ & $\begin{array}{l}0.005 \\
0.004 \\
0.008 \\
0.012 \\
0.004 \\
0.006\end{array}$ \\
\hline $\begin{array}{l}\text { McHardy } \\
\text { Univ. Leicester }\end{array}$ & 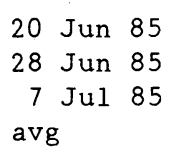 & $\begin{array}{l}3 L x^{10} \\
3 L x \\
3 L x \\
3 L x\end{array}$ & $\begin{array}{l}17.38 \\
17.38 \\
17.38 \\
17.38\end{array}$ & $\begin{array}{r}2^{11} \\
2.2 \\
1.1 \\
1.77\end{array}$ & $\begin{array}{l}-29.53 \\
-29.49 \\
-29.79 \\
-29.58\end{array}$ & $\begin{array}{l}0.18 \\
0.16 \\
0.30 \\
0.13\end{array}$ \\
\hline $\begin{array}{l}\text { Bregman, Glassgold, } \\
\text { Huggins, Kinney } \\
\text { NYU/NRAO }\end{array}$ & $\begin{array}{l}3 \mathrm{Jul} 85 \\
19 \mathrm{Jul} 85\end{array}$ & $\begin{array}{l}2625^{3} \\
2775 \\
2625 \\
2775\end{array}$ & $\begin{array}{l}15.06 \\
15.03 \\
15.06 \\
15.03\end{array}$ & $\begin{array}{l}1.64 \mathrm{E}-16^{4} \\
4.40 \mathrm{E}-16 \\
1.95 \mathrm{E}-16 \\
3.53 \mathrm{E}-16\end{array}$ & $\begin{array}{l}-27.29 \\
-26.82 \\
-27.22 \\
-26.92\end{array}$ & $\begin{array}{l}0.11 \\
0.04 \\
0.18 \\
0.05\end{array}$ \\
\hline $\begin{array}{l}\text { Webb, Smith, Pica, } \\
\text { Pollock, Leacock } \\
\text { Univ. Florida }\end{array}$ & 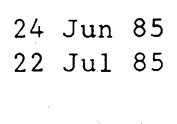 & $\begin{array}{l}4400 \\
4400\end{array}$ & $\begin{array}{l}14.83 \\
14.83\end{array}$ & $\begin{array}{l}18.13^{6} \\
18.88\end{array}$ & $\begin{array}{l}-26.52 \\
-26.82\end{array}$ & $\begin{array}{l}0.07 \\
0.06\end{array}$ \\
\hline UKIRT Service & $19 \mathrm{Ju} 185$ & $1.25^{5}$ & 14.38 & $0.41^{9}$ & -26.37 & 0.0 \\
\hline
\end{tabular}


TABLE 2-Continued

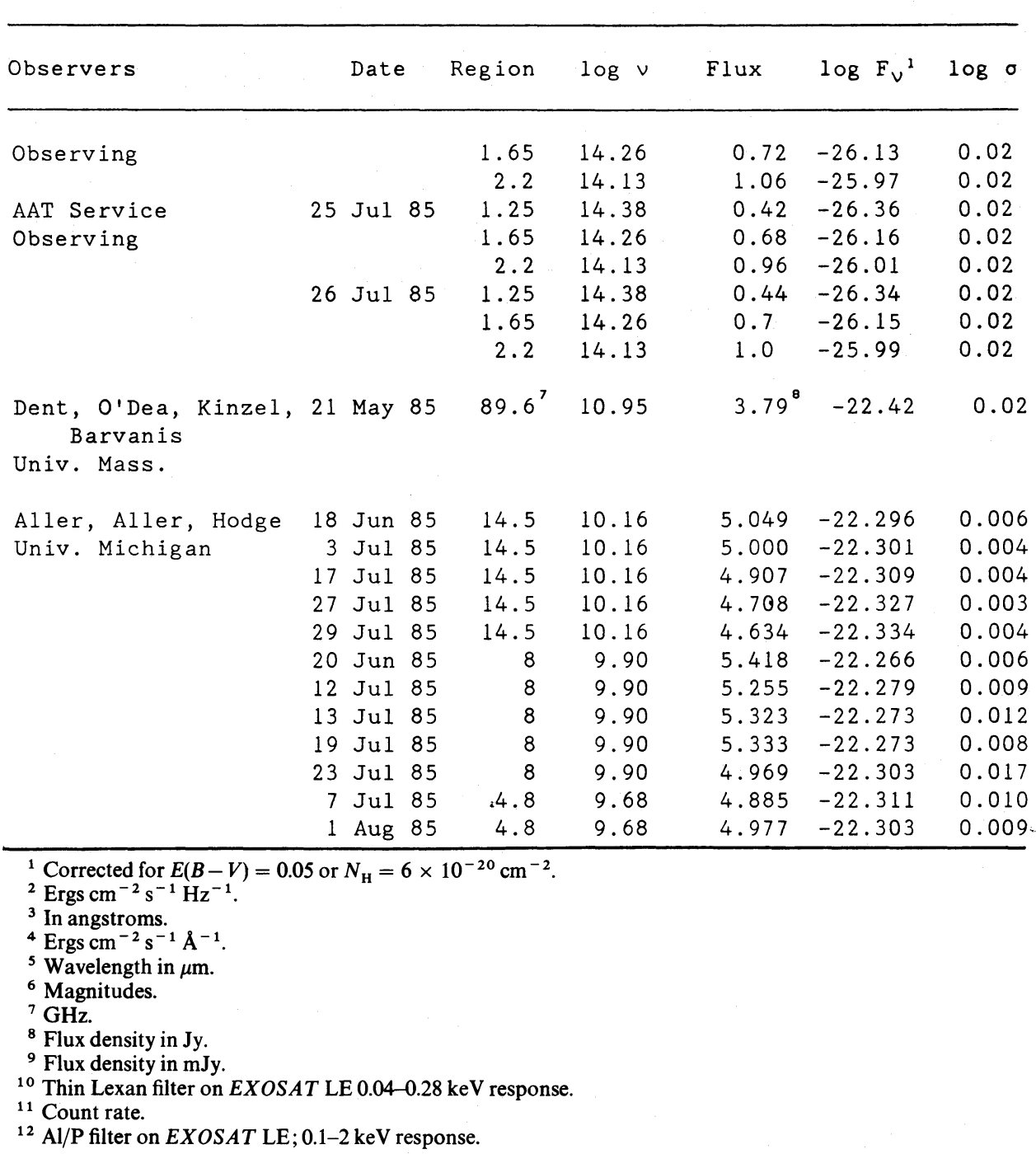

spectra and allow one to distinguish between real changes and those arising from calibration uncertainties.

The radio spectra reveal greater variation at higher frequencies, but the effect is not monotonic. The spectra have a fair amount of structure, such as the local peak near $10<\log$ $v(\mathrm{~Hz})<11$ during 1980 June, and are indicative of optical depth effects. The spectra during 1985 are steeper than the fairly flat spectrum of 1983 December and are typical of a postoutburst spectrum.

The IR-UV spectrum preserves its shape remarkably well despite flux variations of an order of magnitude. The apparent change in shape (and misalignment) between the infrared and optical regions in the 1984 June spectrum is probably due to the infrared data being obtained 8 days after the optical and ultraviolet data. The only definite spectral change in the IR-UV band occurred during 1985 July, when the infrared and optical emission were at their lowest levels. Had the decrease in the ultraviolet flux been as great as that in the infrared, ultraviolet emission would not have been visible with the $I U E$. The optical emission decreased less than the infrared emission but more than the ultraviolet emission. The net effect is that the
1985 July spectrum is significantly less steep (by about $0.6-0.7$ in $\alpha$ ) than other spectra; Oke (1967) also reported a shallower than normal slope at an epoch when 3C 446 was faint. The observed behavior is consistent with the presence of a constant, faint blue bump that is visible only when the source is dim. According to this picture, 3C 446 might resemble an ordinary quasar when it is very faint.

Although spectral variation in $3 \mathrm{C} 446$ is not especially common, it has been observed by other investigators. Our mean optical slope $(\alpha=-1.67$ at $B)$ is the value typically reported by other observers, but Wampler (1967) has reported slopes as shallow as -1.32 and Miller (1981) has reported slopes steeper than -3 (both observations during bright phases). Our mean near-infrared slope $(\alpha=-1.29)$ is close to the values reported by Oke, Neugebauer, and Becklin (1970), Neugebauer et al. (1979), and Allen, Ward, and Hyland (1982), but slopes as steep as $-1.86 \pm 0.06$ are reported by Brown $e t$ al. (1986).

The variation in the $\mathrm{X}$-ray emission is compared with radio and optical-ultraviolet flux density variation in Figure 8. During 1983 December; the X-ray flux decreased by about a 
BREGMAN ET $A L$

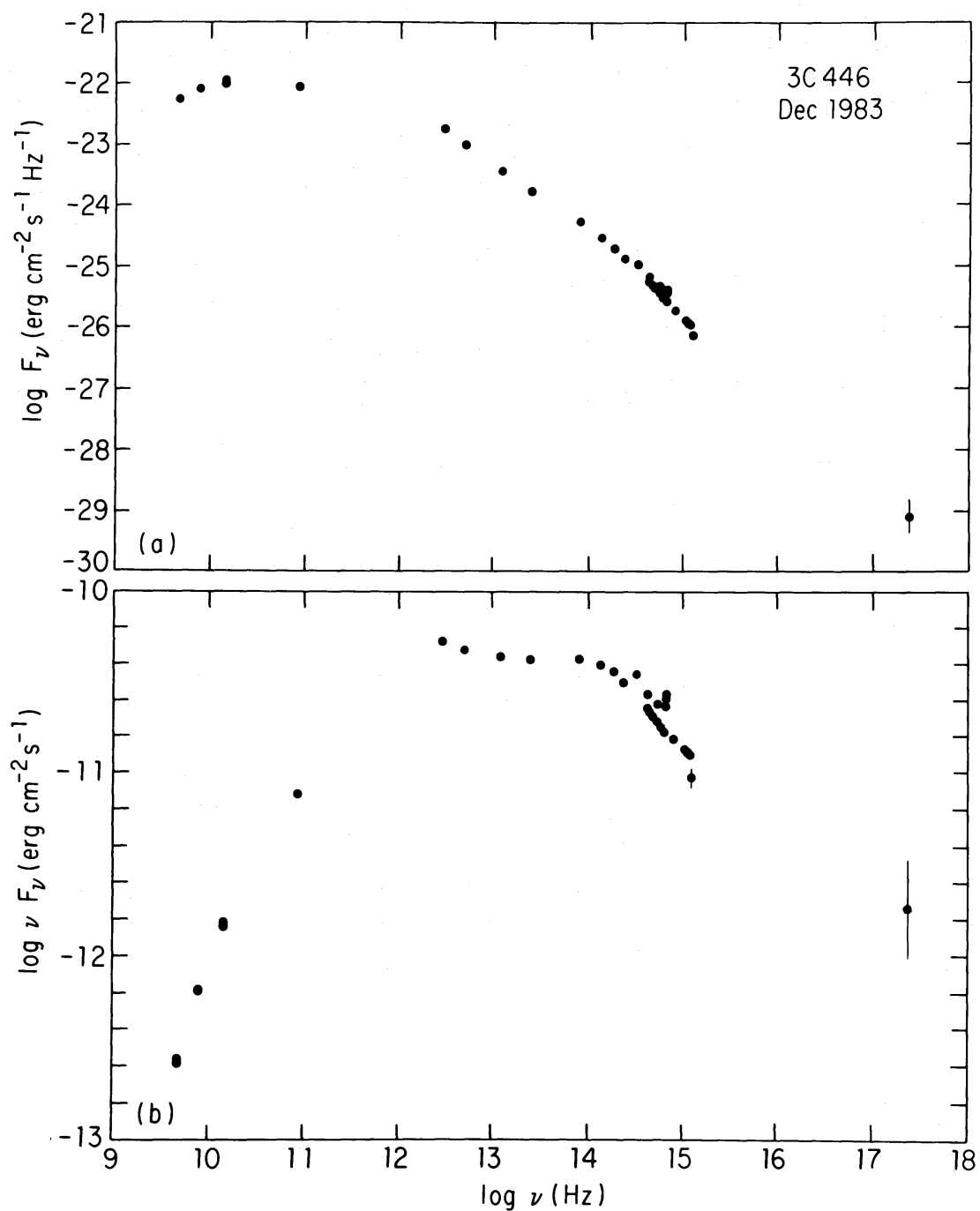

Fig. 6.-The flux density $(a)$ and the power per logarithmic bandwidth $(b)$ are displayed as a function of frequency for the multifrequency spectrum of $3 \mathrm{C} 446$ taken in 1983 December. Errors less than $10 \%$ are not shown. The X-ray emission was variable at this time, and the vertical bar in the X-ray measurement reflects the standard deviation in the average of four measurements.

factor of 3 with no corresponding decrease at optical or radio wavelengths. During the next 19 months, there was considerable decrease in the optical-ultraviolet flux, a trend not found in the radio measurements. The X-ray flux measurements in 1984 May-June are clearly below the mean of the 1983 December data and are consistent with being correlated to the longterm optical-ultraviolet variation. However, the two faintest millimeter wave measurements occurred when the X-ray emission was at its brightest level, which suggests that these wavebands are not closely connected.

\section{c) The Ly $\alpha$ Emission-Line Variation}

Bregman et al. (1986b) have previously reported observations of the $\mathrm{Ly} \alpha$ line in $3 \mathrm{C} 446$. A series of five observations from 1980 June through 1984 October showed that the Ly $\alpha$ line flux changed roughly in proportion to the continuum flux (at nearly constant equivalent width). Because the shortest time scale for the line variation $\left(d t / d \log F_{v}\right)$ is 2 months in the rest frame of $3 \mathrm{C} 446$ (which is probably much less than the distance of the clouds from the central continuum), it was argued (Bregman et al. 1986b) that the emission-line clouds were dis- tributed anisotropically around $3 \mathrm{C} 446$, with a substantial amount of gas lying on the near side of 3C 446, 2-3 pc distant, and close to our line of sight.

In order to better resolve the $\mathrm{Ly} \alpha$ line variation, subsequent IUE observations were carried out in 1985 May-July. The IR-UV continuum was especially faint during this period, with the ultraviolet continuum being 2.3 times fainter than any of our previous measurements. The new measurements are similar to each other, having nearly the same values for both the Ly $\alpha$ flux and the adjacent continuum. These and earlier data are shown in Figure 9; the 1985 data are the four points at the left. At low continuum flux levels, the Ly $\alpha$ flux is independent of the continuum level. It appears to increase at the highest continuum levels measured.

The increase in the equivalent width in $\mathrm{Ly} \alpha$ and the flattening of the spectrum makes 3C 446 seem more like a "normal" quasar. However, even at the faintest levels, when the spectrum was the hardest $[\alpha($ opt-UV $)=-1.25 \pm 0.25$, compared to the normal slope of -2 to -3$]$, it is not yet as flat as normal quasars $(\alpha=-0.6$; Richstone and Schmidt 1980). Although an equivalent width is a useful measure of a line, a 


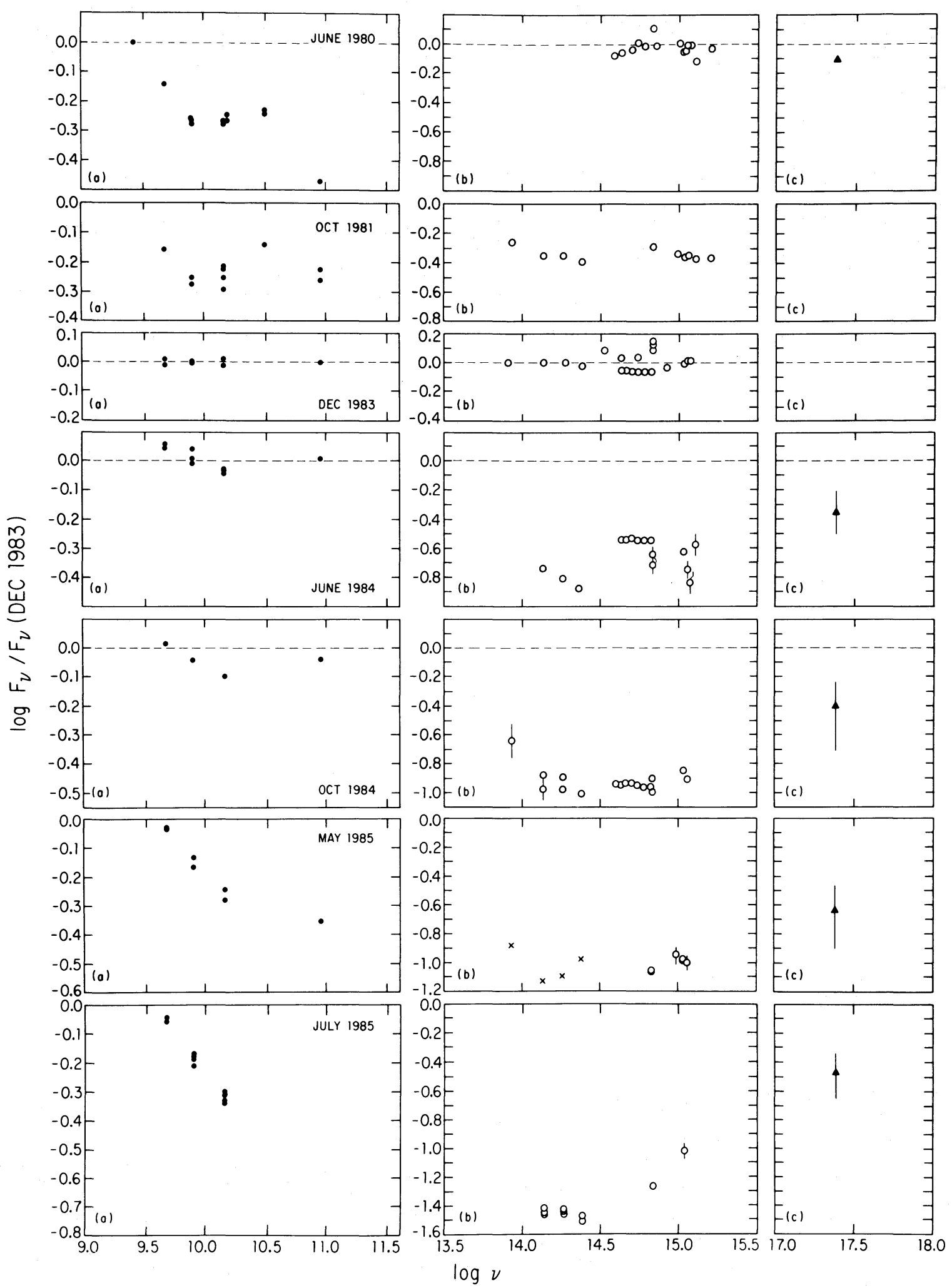

Fig. 7.-Seven multifrequency spectra of 3C 446 normalized to the 1983 December spectrum are displayed in separate radio, infrared-ultraviolet, and X-ray panels. The vertical scale of the radio panel is twice that of the other panels; errors less than $12 \%$ are not shown. The zero line occurs either at the top of each frame or along a dashed line. The infrared data shown in the 1984 June spectrum were obtained about a week apart from the other data, so the relative shift of these data may not be meaningful. 


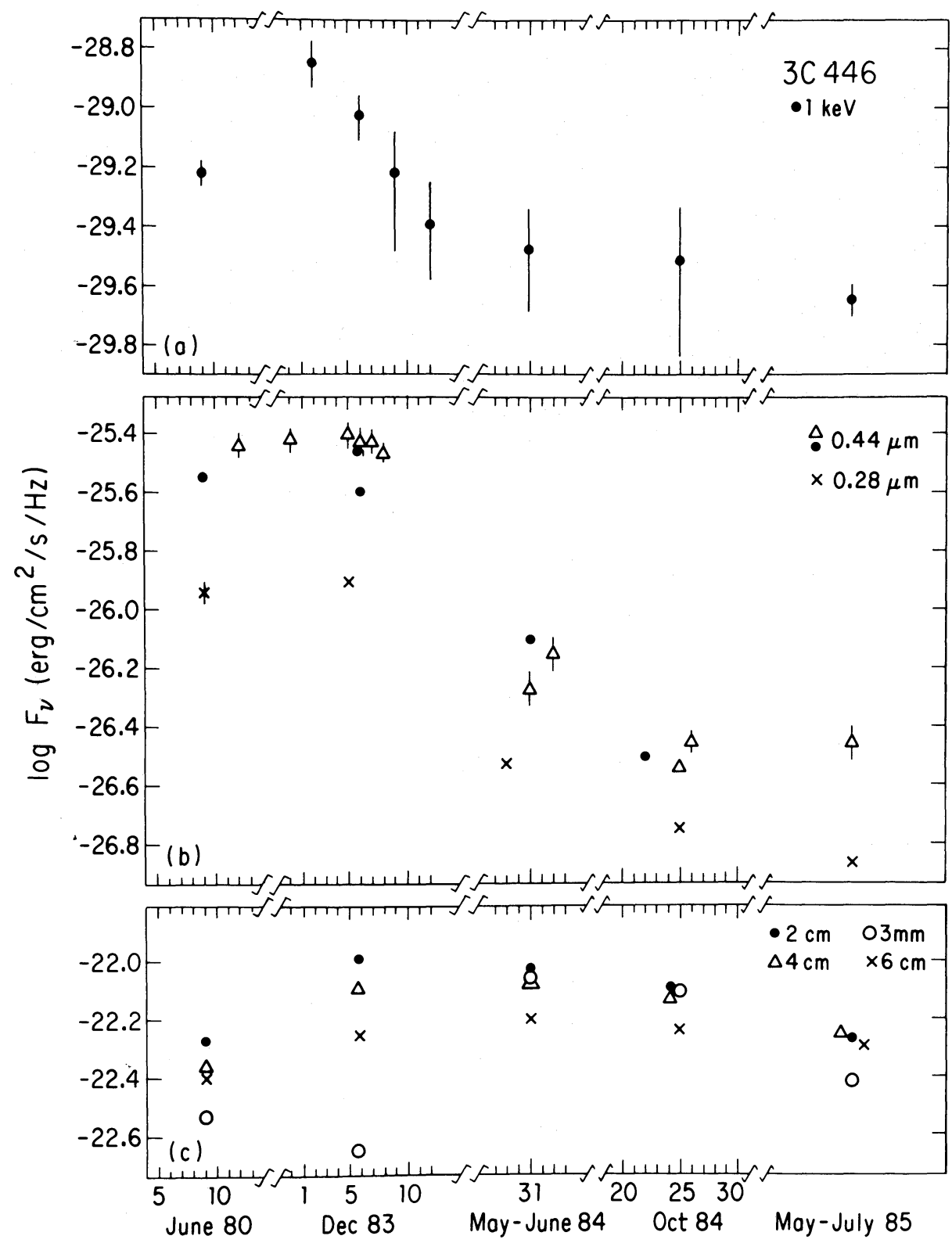

Fig. 8.-X-ray, optical, ultraviolet, and radio fluxes for five epochs. Errors less than $10 \%$ are not shown. The six weak X-ray measurements obtained during 1984 May-July were averaged to improve the signal-to-noise ratio.

physically more significant measure is the percentage of ionizing photons that must be absorbed to produce the observed Ly $\alpha$ strength (the covering factor). If the spectral slope between the optical and ultraviolet bands remains constant at shorter wavelengths, the covering factor in 1985 is $5.1 \% \pm 1.9 \%$, which is similar to its value when the source was brighter $(3.3 \%)$. The nearly constant line fluxes at low optical and ultraviolet light levels may occur because the number of ionizing photons no longer changes due to the presence of a nonvariable ultraviolet blue bump. Alternatively, the lack of Ly $\alpha$ line variation may arise from emission-line gas that does not lie along our line of sight (e.g., spherically distributed clouds) and therefore has a time scale of variability longer than the observing period.

\section{THEORETICAL INTERPRETATION OF CONTINUUM DATA}

The multifrequency data can be usefully interpreted with simple models to obtain estimates of the physical parameters of the emission region. We first discuss the homogeneous synchrotron self-Compton model (see discussion in Bregman et al. 1982, 1984). In applying this model, it is important to identify the frequency at which the synchrotron emitting region first becomes opaque $\left(v_{m}\right)$. The flattening of the spectrum between the millimeter and infrared regions is likely to be due to optical depth effects. Although the turnover region occurs where our data are most incomplete, the data indicate that $v_{m}$ is in the range of $10^{11}-3 \times 10^{12} \mathrm{~Hz}$. Linear extrapolations of the highand low-frequency continua to the point of intersection results 


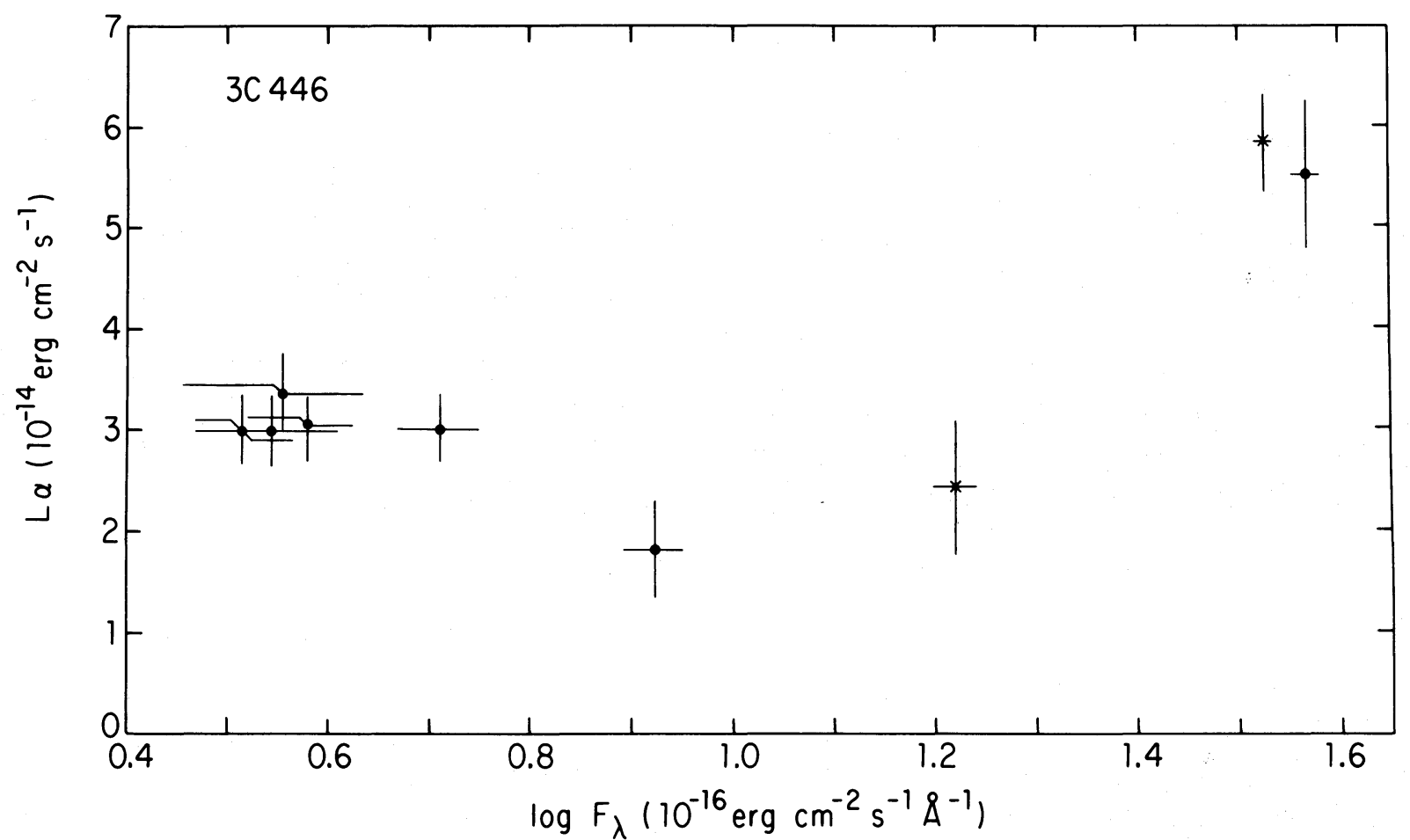

FIG. 9.-The Ly $\alpha$ flux contained in a $30 \AA$ band centered on the line is plotted against the underlying continuum flux density. Data obtained with the LWR are denoted by an $X$, and those obtained with the LWP are denoted by filled circles.

in $v_{m} \approx 3-10 \times 10^{11} \mathrm{~Hz}$. To determine the importance of relativistic boosting, a separate measure for the size of the emitting region at $v_{m}$ is needed. We will assume that such a size is given from the variability time scale (times a relativistic correction factor); the data indicate that the variability time scale at $v_{m}$ is in the range 2-6 months (Table 1). The results of applying the SSC model are given in Table 3 , where several values for $v_{m}$ and the variability time scale $t_{v}$ are used $\left(t_{v}=d t / d \log F_{v}\right)$. We consider the most appropriate models to be $\mathrm{D}$ and $\mathrm{E}$. The best determined quantity is the dimension of the emitting region, $r \approx 1-3 \times 10^{17} \mathrm{~cm}$, while the magnetic field is less well constrained and falls in the range 3-100 G. Although the density appears to be confined to a narrow range $\left(40-100 \mathrm{~cm}^{-3}\right)$, its value depends upon the minimum electron energy (assumed to be $15 \mathrm{MeV}$ ) and the shape of the electron spectrum, which may not be a direct reflection of the slope of the optically thin emission. These difficulties could easily lead to an order of magnitude uncertainty in the density. The models suggest that modest relativistic boosting is required in the emitting plasma, with the Doppler boosting parameter $\delta \approx 2-6$. For the two preferred models (D and E), the particle energy density $\left(3 \times 10^{-3}\right.$ ergs $\left.\mathrm{cm}^{-3}\right)$ is significantly less than the magnetic energy density $\left(1-400 \mathrm{ergs}^{-3}\right)$.

It should be stressed that the above model is a homogeneous SSC model, whereas the variability data suggest that an inhomogeneous model is more appropriate. We have applied the inhomogeneous model of Ghisellini, Maraschi, and Treves (1985) and find that the conditions in the emitting plasma at frequency $v_{m}$ are similar. The important parameters in inhomogeneous models have a power-law dependence in space (e.g., $\left.B \propto r^{-n}\right)$. However, in the majority of these models, most of the optically thin radiation emanates from the same volume, independent of frequency. This leads to variability time scales that are nearly constant throughout the optically thin part of the spectrum (far-IR-UV), in conflict with observations (Table 1). Optically thin radiation at two frequencies may be decoupled if the electron spectrum is not a power-law over a large energy range. In particular, the electron spectrum at some location must have an upper and lower energy cutoff. General inhomogenous models have not been developed, although efforts have been made in this direction (Marscher and Gear 1985).

Despite these shortcomings, a reasonable extrapolation of

TABLE 3

SYNCHROTRON SELF-COMPTON MODELS FOR 3C 446

\begin{tabular}{crcccccr}
\hline \hline Model & $v_{m}(\mathrm{GHz})$ & $F_{v}\left(v_{m}\right)(\mathrm{Jy})$ & $t_{v}($ weeks $)$ & $B(\mathrm{G})$ & $r(\mathrm{pc})$ & $n\left(\mathrm{~cm}^{-3}\right)$ & $\delta$ \\
\hline A $\ldots \ldots .$. & 300 & 6.5 & 6 & 2.5 & 0.067 & 78 & 9.1 \\
B $\ldots \ldots .$. & 1000 & 4. & 6 & 51 & 0.030 & 95 & 4.1 \\
C $\ldots \ldots .$. & 100 & 8. & 13 & 0.31 & 0.17 & 68 & 10.5 \\
D $\ldots \ldots$. & 300 & 6.5 & 13 & 4.8 & 0.089 & 58 & 5.6 \\
E $\ldots \ldots .$. & 1000 & 4. & 13 & 100 & 0.041 & 71 & 2.5 \\
F $\ldots \ldots .$. & 3000 & 1.7 & 13 & 1670 & 0.017 & 147 & 1.1 \\
G $\ldots \ldots .$. & 300 & 6.5 & 26 & 8.9 & 0.116 & 45 & 3.6 \\
H $\ldots \ldots$. & 1000 & 4. & 26 & 184 & 0.053 & 55 & 1.6 \\
\hline
\end{tabular}


the plasma conditions at $v>v_{m}$ can be made. From the variability data, the optical and $\mathrm{X}$-ray region is about an order of magnitude smaller than the region responsible for the radiation near $v_{m}$. Various models (e.g., Marscher 1977; Blandford and Königl 1979) suggest that $B$ and $n$ would be greater in the optical region by factors of 3-30 and 10-100, respectively. This would place the magnetic field in the range of 20-3000 G and the density in the range of $600-6000 \mathrm{~cm}^{-3}$ (models D and E).

An estimate of the conditions in plasma emitting at frequencies below $v_{m}$ is constrained by the flatness of the spectrum and by the VLBI observations. The flatness of the radio spectrum constrains the spatial dependence of $B$ and $n$, and using the model of Ghisellini, Maraschi, and Treves (1985), we find that $B \approx 0.05-3 \mathrm{G}$ and $n \approx 0.3-3 \mathrm{~cm}^{-3}$. The VLBI size of 0.4 mas ( 2 pc; Brown et al. 1981) is the same as the variability size when corrected for $\delta \approx$ few, but the magnetic field deduced from the VLBI data, $1-10 \times 10^{-3} \mathrm{G}$, is less than the theoretical estimate. This disagreement can be resolved if the magnetic field decreases rapidly as a function of radius, or if the relativistic boosting factor becomes increasingly more important for $v<v_{m}$.

It is worth noting that the smallest dimension that we infer, $\sim 10^{16} \mathrm{~cm}$ for the X-ray emitting region, is only an order of magnitude greater than the radius of the last stable orbit around a $10^{9} M_{\odot}$ black hole. Therefore, if the entral engine consists of a massive black hole and an accretion disk, the high-frequency emission may be related to the inner part of the accretion disk.

\section{CONCLUSIONS}

Nearly 20 years of optical and radio monitoring data as well as seven multifrequency spectra (radio through X-ray bands) of the violently variable quasar $3 C 446$ are reported in this paper. The optical monitoring data reveal flickering activity on a time scale of days to weeks and major outbursts that last for a month to a year. The radio data show no comparable flickering but have well-defined outbursts that are of longer duration but lower amplitude than the optical data. The optical and radio data are correlated, with optical outbursts preceding their radio counterparts by 400-600 days. However, a structure function analysis shows that the nature of the variability of the optical $(0.44 \mu \mathrm{m})$ and radio fluxes $(2 \mathrm{~cm})$ are described by different power-law forms, indicating significant processing of the optically emitting plasma before it becomes radio-emitting plasma. In the radio region, outbursts occur progressively later at longer wavelengths, presumably due to optical depth effects. Although the spectral shape in the infrared through X-ray region is usually preserved during brightness variations, there are important exceptions. A well-observed outburst during 1983 occurred more rapidly and with greater amplitude in the optical $(0.44 \mu \mathrm{m})$ than in the infrared region $(1-10 \mu \mathrm{m})$. The variations in the IRAS fluxes at $25-100 \mu \mathrm{m}$ do not closely follow those at the shorter wavelengths, which indicates that a time delay exists between these spectral domains. Also, on a time scale of days, the $\mathrm{X}$-ray emission changes more rapidly and with greater amplitude than the ultraviolet, optical, IR, or radio emission. However, over a time scale of weeks to months, the X-ray and optical-ultraviolet emission are well correlated.

A single-epoch multifrequency spectrum shows that the flat radio continuum turns over at about $3-10 \times 10^{11} \mathrm{~Hz}$ and has a slope $\alpha=-1.1$ in the infrared region. The continuum continues to steepen, reaching a slope of $\alpha=-2$ to -3 in the ultraviolet region. This sharp steepening of the continuum between the infrared and ultraviolet region is reminiscent of the class of objects known as Red Quasars, except the steepening occurs at longer wavelengths in those objects. An extrapolation of the optical-ultraviolet continuum to higher frequencies passes an order of magnitude below the X-ray data, which has a harder spectrum than the optical and ultraviolet continuum. The power is primarily concentrated in the submillimeter and infrared region. The contribution to the power by hard X-ray and gamma-ray emission is not directly observed. However, for reasonable assumptions about the spectrum in this region, the X-ray flux is unlikely to account for more than a quarter of the total flux.

The data suggest that the X-ray emission comes from an emitting region smaller than the regions responsible for the radio through ultraviolet emission. The continuum and variability data are consistent with the $\mathrm{X}$-rays being produced through the inverse Compton process, or some process other than synchrotron emission. The synchrotron self-Compton models suggest that the plasma-emitting radiation near the submillimeter turnover $\left(3-10 \times 10^{11} \mathrm{~Hz}\right)$ has $r \approx 0.4-0.1 \mathrm{pc}$, $B \approx 3-100 \mathrm{G}, n \approx 40-100 \mathrm{~cm}^{-3}$, and a Doppler boosting factor $\delta \approx 2-5$. The variability data indicate that the plasmaemitting optical and ultraviolet radiation has a size about an order of magnitude smaller, which implies that the magnetic field is about 10-30 times greater, and the density is about $30-100$ times greater (assuming the same value for $\delta$ ). The flat radio spectrum and the VLBI observations (Brown et al. 1981) indicate that the magnetic field and density decrease (by two orders of magnitude) and the size increases (by about an order of magnitude) relative to the plasma emitting in the $3-10 \times 10^{11} \mathrm{~Hz}$ region.

In a continuation of the Ly $\alpha$ line variation study (Bregman et al. 1985), we find that the Ly $\alpha$ line flux scales with the continuum at high but not at low brightness levels. The spectrum hardens as the source dims, which would occur if a nonvariable blue bump were present. A nonvariable blue bump would provide a minimum number of ionizing photons, which would lead to a minimum emission line flux, as is observed.

We thank Martin Elvis, who was responsible for scheduling the Einstein X-ray observations simultaneously with other observations. IUE observing at NYU was supported by NASA grant NAG 5-73; McHardy thanks the SERC and the X-ray group at Leicester for their support; radio astronomy at University of Michigan is supported by the NSF; Miller and Stephens wish to acknowledge Lick Observatory and the NSF for their support; Dent, Balonek, and Barvainis have been aided by the NSF and the University of Massachusetts; infrared astronomy at Caltech gratefully acknowledges continued support by the NSF; funding for IRAS and data reduction was provided by NASA; Wiśniewski would like to thank Steward Observatory for their support; observing at the University of Florida has been supported by continuing grants from the NSF, the current grant being AST-8516269. 


\section{REFERENCES}

Allen, D. A., Ward, M. J., and Hyland, A. R. 1982, M.N.R.A.S., 199, 969.

Aller, H. D., Aller, M. F., Latimer, G. E., and Hodge, P. E. 1985, Ap. J. Suppl., $59,513$.

Balonek, T. J. 1982, Ph.D. thesis, University of Massachusetts.

Barbieri, C., Cristiani, S., Omizzolo, S., and Romano, G. 1985, Astr. Ap., 142, 316.

Blandford, R. D., and Königl, A. 1979, Ap. J., 232, 34.

Bregman, J. N., Lebofsky, M. J., Aller, M. F., Rieke, G. H., Aller, H. D., Hodge, P. E., Glassgold, A. E., and Huggins, P. J. 1981, Nature, 293, 714.

Bregman, J. N., et al. 1982, Ap. J., 253, 19.

. 1984, Ap. J., 276, 454.

Bregman, J. N., Glassgold, A. E., Huggins, P. J., and Kinney, A. L. 1986b, Ap. J., 301, 698 .

Brown, L. M. J., Robson, E. I., Gear, W. K., Crosthwaite, R. P., McHardy, I. M., Hanson, C. G., Geldzahler, B. J., and Webb, J. R. 1986, M.N.R.A.S., 219,671.

Brown, R. L., Johnston, K. J., Briggs, F. H., Wolfe, A. M., Neff, S. G., and Walker, R. C. 1981, Ap. Letters, 21, 105.

Burstein, D., and Heiles, C. 1978, Ap. J., 225, 40.

Courvoisier, T. J.-L., Bell-Burnell, J., and Blecha, A. 1986, Astr. Ap., 169, 43.

Garilli, B., and Tagliaferri, G. 1986, Ap. J, 301, 703.

Ghisellini, G., Maraschi, L., and Treves, A. 1985, Astr. Ap., 146, 204.

Glassgold, A. E., et al. 1983, Ap. J., 274, 101.
Kardashev, N. S. 1962, Soviet Astr.-AJ, 6, 317.

Marscher, A. P. 1977, Ap. J., 216, 244.

Marscher, A. P., and Gear, W. K. 1985, Ap. J., 298, 114

Myers, T., and Spangler, S. R. 1985, Ap. J., 291, 52.

Miller, H. R. 1981, Ap.J., 244, 426.

Miller, J. S., and French, H. B. 1978, in Pittsburgh Conference On BL Lac Objects, ed. A. M. Wolfe (Pittsburgh: University of Pittsburgh), p. 228.

Moore, R. L., and Stockman, H. S. 1984, Ap. J., 279, 485.

Neugebauer, G., Miley, G. K., Soifer, B. T., and Clegg, P. E. 1986, Ap. J., 308, 815 .

Neugebauer, G., Oke, J. B., Becklin, E. E., and Matthews, K. 1979, Ap. J., 230, 79.

Oke, J. B. $1967, A p . J ., 147,901$

Oke, J. B., Neugebauer, G., and Becklin, E. E. 1970, Ap. J., 159, 341

Pacholczyk, A. G. 1970, Radio Astrophysics (San Francisco: Freeman), p. 87

Richstone, D. O., and Schmidt, M. 1980, Ap. J., 235, 361.

Rothschild, R. E., Mushotzky, R. F., Baity, W. A., Gruber, D. E., Matteson, J. L., and Peterson, L. E. 1983, Ap. J., 269, 423.

Simon, R. S., Johnston, K. J., and Spencer, J. H. 1985, Ap. J., 290, 66

Simonetti, J. H., Cordes, J. M., and Heeschen, D. S. 1985, Ap. J., 296, 46.

Stephens, S. A., and Miller, J. S. 1984, Bull. AAS, 16, 1007.

Urry, C. M. 1986, talk given at meeting on Supermassive Black Holes.

Wampler, E. J. 1967, Ap. J. (Letters), 148, L101.

Wills, B. J., et al. 1983, Ap. J., 274, 62.

H. D. Aller, M. F. Aller, and P. E. Hodge: Department of Astronomy, University of Michigan, 953 Physics-Astronomy Building, Ann Arbor, MI 48109

T. J. BALONEK : Department of Physics and Astronomy, Colgate University, Hamilton, NY 11346

R. BARVAINIS: Haystack Observatory, NEROC, Route 40, Westford, MA 01886

J. N. Bregman: National Radio Astronomy Observatory, Edgemont Road, Charlottesville, VA 22901

W. A. Dent: Department of Physics and Astronomy, GR Tower B, University of Massachusetts, Amherst, MA 01003

J. H. Elias: CTIO, NOAO, P.O. Box 26732, Tucson, AZ 85726

A. E. Glassgold and P. J. Huggins: Physics Department, New York University, 4 Washington Place, New York, NY 10003

C. D. IMPEY and W. Z. WiśNIEWSKI: Steward Observatory, University of Arizona, Space Sciences Building, Tucson, AZ 85721

A. L. KInNEY: Space Telescope Science Institute, Homewood Campus, The Johns Hopkins University, Baltimore, MD 21218

R. J. LeACoCK, A. G. Smith, and J. R. WebB: Department of Astronomy, University of Florida, Gainesville, FL 32611

K. Matthews, G. Neugebauer, and B. T. Solfer: Division of Physics, Mathematics, and Astronomy, Caltech, Pasadena, CA 91125

I. McHardy: X-Ray Astronomy Group, Department of Physics, University of Leicester, University Road, Leicester, LE1 7RH, England

J. S. Miller and S. A. StePhens: Lick Observatory, University of California, Santa Cruz, CA 95064

A. J. PICA: Department of Physical Sciences, Salisbury State College, Salisbury, MD 21801

J. T. Pollock: Department of Physics and Astronomy, Appalachian State University, Boone, NC 28608 